\title{
Positive Periodic Solutions for Impulsive Functional Differential Equations with Infinite Delay and Two Parameters
}

\author{
Zhenguo Luo, ${ }^{1,2}$ Liping Luo, ${ }^{1}$ and Yunhui Zeng ${ }^{1}$ \\ ${ }^{1}$ Department of Mathematics, Hengyang Normal University, Hengyang, Hunan 421008, China \\ ${ }^{2}$ Department of Mathematics, National University of Defense Technology, Changsha 410073, China
}

Correspondence should be addressed to Zhenguo Luo; robert186@163.com

Received 29 June 2013; Accepted 2 October 2013; Published 5 January 2014

Academic Editor: Meng Fan

Copyright (c) 2014 Zhenguo Luo et al. This is an open access article distributed under the Creative Commons Attribution License, which permits unrestricted use, distribution, and reproduction in any medium, provided the original work is properly cited.

\begin{abstract}
We apply the Krasnoselskii's fixed point theorem to study the existence of multiple positive periodic solutions for a class of impulsive functional differential equations with infinite delay and two parameters. In particular, the presented criteria improve and generalize some related results in the literature. As an application, we study some special cases of systems, which have been studied extensively in the literature.
\end{abstract}

\section{Introduction}

First, we give the following definitions. Let $J \subset R$ denote by $P C\left(J, R^{n}\right)$ the set of operators $\varphi: J \rightarrow R^{n}$ which are continuous for $t \in J, t \neq t_{k}$ and have discontinuities of the first kind at the points $t_{k} \in J\left(k \in Z_{+}\right)$but are continuous from the left at these points. For each $y=\left(y_{1}, y_{2}, \ldots, y_{n}\right)^{T} \in R^{n}$, the norm of $y$ is defined as $|y|=\sum_{i=1}^{n}\left|y_{i}\right|$. The matrix $A>$ $B(A \leq B)$ means that each pair of corresponding elements of $A$ and $B$ satisfies the inequality " > " " $\leq$ "). In particular, $A$ is called a positive matrix if $A>0$.

Impulsive differential equations are suitable for the mathematical simulation of evolutionary process whose states are subject to sudden changes at certain moments. Equations of this kind are found in almost every domain of applied sciences; numerous examples are given in [1-3]. In recent years, in [4-11], many researchers have obtained some properties of impulsive differential equations, such as oscillation, asymptotic behavior, stability, and existence of solutions. However, to this day, still no scholars investigate the existence of multiple positive periodic solutions for impulsive functional differential equations with infinite delay and two parameters. Motivated by this, in this paper, we mainly consider the following impulsive functional differential equations with two parameters:

$$
\begin{gathered}
y^{\prime}(t)=-A(t, y(t)) y(t)+\lambda B(t, y(t)) F(t, u(t)), \\
t \in R, t \neq t_{k}, \\
\Delta y\left(t_{k}\right)=\mu I_{k}\left(t_{k}, y\left(t_{k}\right)\right), \quad k \in Z_{+}, \\
y^{\prime}(t)=A(t, y(t)) y(t)-\lambda B(t, y(t)) F(t, u(t)), \\
t \in R, t \neq t_{k}, \\
\Delta y\left(t_{k}\right)=\mu I_{k}\left(t_{k}, y\left(t_{k}\right)\right), \quad k \in Z_{+},
\end{gathered}
$$

where

$$
\begin{gathered}
u(t)=\left(y\left(c_{1}(t)\right), \ldots, y\left(c_{n-1}(t)\right),\right. \\
\left.\int_{-\infty}^{t} k(t-\xi) y(\xi) d \xi\right),
\end{gathered}
$$

and $\lambda>0, \mu>0$ are two parameters, $A(t, y(t))=$ $\operatorname{diag}\left[a_{1}(t, y(t)), a_{2}(t, y(t)), \ldots, a_{n}(t, y(t))\right], B(t, y(t))=$ $\operatorname{diag}\left[b_{1}(t, y(t)), b_{2}(t, y(t)), \ldots, b_{n}(t, y(t))\right], a_{i}, b_{i} \in C\left(R \times R^{+}\right.$, $\left.R^{+}\right)(i=1,2, \ldots, n)$ are $\omega$-periodic, that is, $a_{i}(t+\omega, y(t+\omega))=$ 
$a_{i}(t, y(t)), b_{i}(t+\omega, y(t+\omega))=b_{i}(t, y(t)), F=\left(f_{1}, \ldots, f_{n}\right)^{T}$, $F(t, u(t))$ is an operator on $R \times B C\left(R, R^{n}\right)$ (here $B C\left(R, R^{n}\right)$ denotes the Banach space of bounded continuous operator $\phi: R \rightarrow R^{n}$ with the norm $\|\phi\|=\sum_{i+1}^{n} \sup _{\theta \in R}|\phi(\theta)|$, where $\left.\phi=\left(\phi_{1}, \ldots, \phi_{n}\right)^{T}\right), f_{i}(t+\omega, u(t+\omega))=f_{i}(t, u(t))$, $\Delta y\left(t_{k}\right)=y\left(t_{k}^{+}\right)-y\left(t_{k}\right)$ (here $y\left(t_{k}^{+}\right)$represents the right limit of $y(t)$ at the point $\left.t_{k}\right), I_{k}=\left(I_{1 k}, I_{2 k}, \ldots, I_{n k}\right) \in C\left(R_{+}^{n}, R_{-}^{n}\right)$, that is, $y(t)$ changes decreasingly suddenly at times $t_{k} . \omega>0$ is a constant, $Z_{+}=\{1,2,3, \ldots\}, R=(-\infty,+\infty), R_{+}=[0,+\infty)$, and $R_{-}=(-\infty, 0]$. We assume that there exists an integer $q>0$ such that $t_{k+q}=t_{k}+\omega, I_{i(k+q)}=I_{i k}, i=1,2, \ldots, n$, where $0<t_{1}<t_{2}<\cdots<t_{q}<\omega$.

Models of forms (1) and (2) have been proposed for population dynamics (single species growth models), physiological processes (such as production of blood cells, respiration, and cardiac arrhythmias), and other practical problems. Equations (1) and (2) are very general and incorporate many famous mathematical models extensively studied in the literature [12-21]. In this paper, we will study the existence of positive periodic solutions in more cases than the previously mentioned papers and obtain some easily verifiable sufficient criteria. tions.

Throughout the paper, we make the following assump-

$\left(H_{1}\right) \quad a_{i}, b_{i}: R \times R_{+} \rightarrow R_{+}$satisfy Caratheodory conditions; that is, $a_{i}(t, y)$ and $b_{i}(t, y)$ are locally Lebesgue measurable in $t$ for each fixed $y$ and are continuous in $y$ for each fixed $t$ are $\omega$-periodic functions in $t$. Moreover, there exist $\omega$-periodic functions $a_{1 i}, a_{2 i}, b_{1 i}, b_{2 i}: R \rightarrow R+$, which are locally bounded Lebesgue measurable so that $a_{1 i}(t) \leq a_{i}(t, y(t)) \leq$ $a_{2 i}(t), b_{1 i}(t) \leq b_{1 i}(t, y(t)) \leq b_{2 i}(t)$ and $\int_{0}^{\omega} a_{1 i}(t) d t>0$, $\int_{0}^{\omega} b_{1 i}(t) d t>0 . \lambda>0, \mu>0$ are two parameters.

$\left(H_{2}\right) f_{i}(t, u(t))$ is $\omega$-periodic with respect to the first variable, that is, $f_{i}(t+\omega, u(t+\omega))=f_{i}(t, u(t))$ such that $f_{i}(t, u(t)) \neq \equiv, i=1,2, \ldots, n$.

$\left(H_{3}\right)$ The delay kernel $k: R_{+} \rightarrow R_{+}$is integrable and is normalized such that $\int_{0}^{+\infty} k(t) d t=1, c_{j}(t): R \rightarrow R$ such that $c_{j}(t) \leq t, j=1,2, \ldots, n-1$.

$\left(H_{4}\right)\left\{t_{k}\right\}, k \in Z_{+}$satisfies $0<t_{1}<t_{2}<\cdots<t_{k}<$ $\cdots$, and $\lim _{k \rightarrow+\infty} t_{k}=+\infty ; I_{k}: R \times R_{+} \rightarrow R$, $k \in Z_{+}$satisfy Caratheodory conditions and are $\omega$ periodic functions in $t$. Moreover, $I_{k}(t, 0)=0$ for all $k \in Z^{+}$. There exists a positive constant $q$ such that $t_{k+q}=t_{k}+\omega, I_{k+q}\left(t_{k+q}, y\left(t_{k+q}\right)\right)=I_{k}\left(t_{k}, y\left(t_{k}\right)\right), k \in Z_{+}$. Without loss of generality, we can assume that $t_{k} \neq 0$ and $[0, \omega] \cap\left\{t_{k}, k \in Z^{+}\right\}=\left\{t_{1}, t_{2}, \ldots, t_{q}\right\}$.

In addition, the parameters in this paper are assumed to be not identically equal to zero.

To conclude this section, we summarize in the following a few concepts and results that will be needed in our arguments.

Definition 1 (see [22]). Let $X$ be a real Banach space and let $E$ be a closed, nonempty subset of $X . E$ is said to be a cone if

(1) $\alpha x+\beta y \in E$ for all $x, y \in E$, and $\alpha, \beta>0$;

(2) $x,-x \in E$ imply $x=0$.
Lemma 2 (see Krasnoselskii's fixed point theorem [23-26]). Let $E$ be a cone in a real Banach space $X$. Assume that $\Omega_{1}$ and $\Omega_{2}$ are open subsets of $X$ with $0 \in \Omega_{1} \subset \overline{\Omega_{1}} \subset \Omega_{2}$, where $\Omega_{i}=\left\{x \in X:\|x\|<r_{i}\right\}(i=1,2)$. Let $T: E \cap\left(\Omega_{2} \backslash \overline{\Omega_{1}}\right) \rightarrow E$ be a completely continuous operator and satisfies either

(1) $\|T x\| \geq\|x\|$, for any $x \in E \cap \partial \Omega_{1}$ and $\|T x\| \leq\|x\|$, for any $x \in E \cap \partial \Omega_{2}$,

or

(2) $\|T x\| \leq\|x\|$, for any $x \in E \cap \partial \Omega_{1}$ and $\|T x\| \geq\|x\|$, for any $x \in E \cap \partial \Omega_{2}$.

\section{Then, $T$ has a fixed point in $E \cap\left(\Omega_{2} \backslash \overline{\Omega_{1}}\right)$.}

For convenience in the following discussion, we introduce the following notations:

$$
\begin{aligned}
& F^{a}=\limsup _{u \in E,\|u\| \rightarrow a} \max _{t \in[0, \omega]} \frac{\int_{0}^{\omega}|F(t, u(t))| d t}{\|u\|}, \\
& F_{a}=\liminf _{u \in E,\|u\| \rightarrow a} \min _{t \in[0, \omega]} \frac{\int_{0}^{\omega}|f(t, u(t))| d t}{\|u\|}, \\
& I^{a}=\limsup _{y \in E,\|y\| \rightarrow a} \max _{t \in[0, \omega]} \frac{\sum_{t \leq t_{k}<t+\omega\left|I_{k}(t, y)\right|}^{\|y\|},}{\| y \mid}, \\
& I_{a}=\liminf _{y \in E,\|y\| \rightarrow a} \min _{t \in[0, \omega]} \frac{\sum_{t \leq t_{k}<t+\omega}\left|I_{k}(t, y)\right|}{\|y\|}
\end{aligned}
$$

where $a$ denotes either 0 or $\infty,\|u\|=\max \left\{\left|u_{1}\right|,\left|u_{2}\right|, \ldots,\left|u_{n}\right|\right\}$, and $\|y\|=\max \left\{\left|y_{1}\right|,\left|y_{2}\right|, \ldots,\left|y_{n}\right|\right\}$.

The paper is organized as follows. In Section 2, firstly, we give some definitions and lemmas. Secondly, we derive some existence theorems for one or two positive periodic solutions of (1) which are established by using Krasnoselskii's fixed point theorem under some conditions. In Section 3, existence theorems for one or two positive periodic solutions of (2) are also established by using Krasnoselskii's fixed point theorem under some conditions. As applications in Section 4, we study some particular cases of systems (1) and (2) which have been investigated extensively in the references mentioned earlier.

\section{Existence of Periodic Solution of (1)}

We establish the existence of positive periodic solutions of (1) by applying the Krasnoselskii's fixed point theorem on cones. We will first make some preparations and list below a few preliminary results. For $(t, s) \in R^{2}, 1 \leq i \leq n$, we define

$$
\begin{gathered}
G_{i}(t, s)=\frac{e^{\int_{t}^{s} a_{i}(\xi, y(\xi))} d \xi}{e^{\int_{0}^{\omega} a_{i}(\xi, y(\xi))} d \xi-1}, \\
G(t, s)=\operatorname{diag}\left[G_{1}(t, s), G_{2}(t, s), \ldots, G_{n}(t, s)\right] .
\end{gathered}
$$


It is clear that $G_{i}(t+\omega, s+\omega)=G_{i}(t, s),\left(\partial G_{i}(t, s) / \partial t\right)=$ $a_{i}(t, y(t)) G_{i}(t, s), G_{i}(t, t+\omega)-G_{i}(t, t)=1$. In view of $\left(H_{1}\right)$, we also define for $1 \leq i \leq n$

$$
\begin{gathered}
\alpha_{i}:=\min _{0 \leq t \leq s \leq \omega}\left|G_{i}(t, s)\right|=\frac{1}{e^{\int_{0}^{\omega} a_{2 i}(\xi) d \xi}-1}, \\
\beta_{i}:=\max _{0 \leq t \leq s \leq \omega}\left|G_{i}(t, s)\right|=\frac{e^{\int_{0}^{\omega} a_{2 i}(\xi) d \xi}}{e^{\int_{0}^{\omega} a_{1 i}(\xi) d \xi}-1}, \\
\alpha=\min _{1 \leq i \leq n} \alpha_{i}, \quad \beta=\max _{1 \leq i \leq n} \beta_{i}, \quad \sigma=\frac{\alpha}{\beta} \in(0,1), \\
B_{i}(t)=\max \left\{\left|b_{1 i}(t)\right|,\left|b_{2 i}(t)\right|\right\}, \\
B_{i}^{\prime}(t)=\min \left\{\left|b_{1 i}(t)\right|,\left|b_{2 i}(t)\right|\right\}, \\
B(t)=\max _{1 \leq i \leq n}\left\{B_{i}(t)\right\}, \quad B^{\prime}(t)=\min _{1 \leq i \leq n}\left\{B_{i}^{\prime}(t)\right\} .
\end{gathered}
$$

Let $X=\left\{y=\left(y_{1}(t), y_{2}(t), \ldots, y_{n}(t)\right)^{T} \in P C\left(R, R^{n}\right)\right.$ । $y(t+\omega)=y(t)\}$ with the norm $\|y\|=\sum_{i=1}^{n}\left|y_{i}\right|_{0},\left|y_{i}\right|_{0}=$ $\sup _{t \in[0, \omega]}\left|y_{i}(t)\right|$. It is easy to verify that $(X,\|\cdot\|)$ is a Banach space. Define $E$ as a cone in $X$ by

$$
\begin{array}{r}
E=\left\{y=\left(y_{1}(t), y_{2}(t), \ldots, y_{n}(t)\right)^{T} \in X: y_{i}(t) \geq \sigma\left\|y_{i}\right\|_{0},\right. \\
t \in[0, \omega]\} .
\end{array}
$$

We easily verify that $E$ is a cone in $X$. We define an operator $T: X \rightarrow X$ as follows:

$$
(T y)(t)=\left(\left(T_{1} y\right)(t),\left(T_{2} y\right)(t), \ldots,\left(T_{n} y\right)(t)\right)^{T},
$$

where

$$
\begin{aligned}
\left(T_{i} y\right)(t)= & \lambda \int_{t}^{t+\omega} G_{i}(t, s) b_{i}(s, y(s)) f_{i}(s, u(s)) d s \\
& +\mu \sum_{t \leq t_{k}<t+\omega} G_{i}\left(t, t_{k}\right) I_{i k}\left(t_{k}, y\left(t_{k}\right)\right) .
\end{aligned}
$$

The proofs of the main results in this paper are based on an application of Krasnoselskii's fixed point theorem in cones. To make use of the fixed point theorem in cone, firstly, we need to introduce some definitions and lemmas.

Definition 3 (see [1]). A function $y: R \rightarrow(0,+\infty)$ is said to be a positive solution of (1) if the following conditions are satisfied:

(a) $y(t)$ is absolutely continuous on each $\left(t_{k}, t_{k+1}\right)$;

(b) for each $k \in Z_{+}, y\left(t_{k}^{+}\right)$and $y\left(t_{k}^{-}\right)$exist, and $y\left(t_{k}^{-}\right)=$ $y\left(t_{k}\right)$

(c) $y(t)$ satisfies the first equation of (1) for almost everywhere in $R$ and $y\left(t_{k}\right)$ satisfies the second equation of (1) at impulsive point $t_{k}, k \in Z_{+}$.

Definition 4 (see [22]). Let $X$ be a real Banach space; $E$ is a cone of $X$. The semiorder induced by the cone $E$ is denoted by " $\leq$ ". That is, $x \leq y$ if and only if $y-x \in P$ for any $x, y \in E$.
Lemma 5 (see [27]). Assume that $f(t)$ and $g(t)$ are continuous nonnegative functions defined on the interval $[\alpha, \beta]$; then there exists $\xi \in[\alpha, \beta]$ such that

$$
\int_{\alpha}^{\beta} f(t) g(t) d t=f(\xi) \int_{\alpha}^{\beta} g(t) d t .
$$

Lemma 6. Assume that $\left(H_{1}\right)-\left(H_{4}\right)$ hold. The existence of positive $\omega$-periodic solution of (1) is equivalent to that of nonzero fixed point of $T$ in $E$.

Proof. Assume that $y=\left(y_{1}(t), y_{2}(t), \ldots, y_{n}(t)\right)^{T} \in X$ is a periodic solution of (1). Then, we have

$$
\begin{aligned}
& {\left[y_{i}(t) e^{\int_{0}^{t} a_{i}(s, y(s)) d s}\right]^{\prime}} \\
& =\lambda e^{\int_{0}^{t} a_{i}(s, y(s)) d s} b_{i}(t, y(t)) f_{i}(t, u(t)), \\
& \quad t \neq t_{k}, i=1,2, \ldots, n .
\end{aligned}
$$

Integrating the above equation over $[t, t+\omega]$, we can have

$$
\begin{aligned}
& \left.y_{i}(v) e^{\int_{0}^{v} a_{i}(s, y(s)) d s}\right|_{t} ^{t_{m_{1}}+n \omega} \\
& +\left.y_{i}(v) e^{\int_{0}^{v} a_{i}(s, y(s)) d s}\right|_{t_{m_{1}}+n \omega} ^{t_{m_{2}}+n \omega} \\
& +\cdots+\left.y_{i}(v) e^{\int_{0}^{v} a_{i}(s, y(s)) d s}\right|_{t_{m_{q}}+n \omega} ^{t+\omega} \\
& =\lambda \int_{t}^{t+\omega} e^{\int_{0}^{v} a_{i}(s, y(s)) d s} b_{i}(v, x(v)) f_{i}(v, u(v)) d v,
\end{aligned}
$$

where $t_{m_{k}}+n \omega \in(t, t+\omega), m_{k} \in\{1,2, \ldots, q\}, k=1,2, \ldots, q$, $n \in Z_{+}$.

Therefore,

$$
\begin{aligned}
& y_{i}(t) e^{\int_{0}^{t} a_{i}(s, y(s)) d s}\left[e^{\int_{t}^{t+\omega} a_{i}(s, y(s)) d s}-1\right] \\
& -\sum_{t \leq t_{k}<t+\omega} \Delta y_{i}\left(t_{m_{k}}\right) e^{\int_{0}^{t_{m_{k}}+n \omega}} a_{i}(s, y(s)) d s \\
& =\lambda \int_{t}^{t+\omega} e^{\int_{0}^{v} a_{i}(s, y(s)) d s} b_{i}(v, y(v)) f_{i}(v, u(v)) d v,
\end{aligned}
$$

which can be transformed into

$$
\begin{aligned}
y_{i}(t)= & \lambda \int_{t}^{t+\omega} G_{i}(t, s) b_{i}(s, y(s)) f_{i}(s, u(s)) d s \\
& +\mu \sum_{t \leq t_{k}<t+\omega} G_{i}\left(t, t_{k}\right) I_{i k}\left(t_{k}, y\left(t_{k}\right)\right) \\
= & \left(T_{i} y\right)(t) .
\end{aligned}
$$

Thus, $y_{i}$ is a periodic solution for (9). 
If $y=\left(y_{1}(t), y_{2}(t), \ldots, y_{n}(t)\right)^{T} \in X$ and $T y=\left(T_{1} y\right.$, $\left.T_{2} y, \ldots, T_{n} y\right)^{T}=y$ with $y \neq 0$, then for any $t=t_{k}$, derivative the two sides of (9) about $t$,

$$
\begin{aligned}
\left(T_{i} y\right)^{\prime}(t) & \\
=\frac{d}{d t} & {\left[\lambda \int_{t}^{t+\omega} G_{i}(t, s) b_{i}(s, y(s)) f_{i}(s, u(s)) d s\right] } \\
=\lambda & {\left[G_{i}(t, t+\omega) b_{i}(t+\omega, y(t+\omega)) f_{i}(t+\omega, u(t+\omega))\right.} \\
& \left.\quad-G_{i}(t, t) b_{i}(t, y(t)) f_{i}(t, u(t))\right] \\
& -a_{i}(t, y(t)) y_{i}(t) \\
= & -a_{i}(t, y(t)) y_{i}(t)+\lambda b_{i}(t, y(t)) f_{i}(t, u(t)) \\
= & y_{i}^{\prime}(t) .
\end{aligned}
$$

For any $t=t_{j}, j \in Z_{+}$, we have from (9) that

$$
\begin{aligned}
y_{i}\left(t_{j}^{+}\right)-y_{i}\left(t_{j}\right)= & \lambda \int_{t_{j}}^{t_{j}+\omega}\left[G_{i}\left(t_{j}^{+}, s\right)-G_{i}\left(t_{j}, s\right)\right] \\
& \times b_{i}(s, y(s)) f_{i}(s, u(s)) d s \\
& +\mu \sum_{t_{j}^{+} \leq t_{k}<t_{j}+\omega} G_{i}\left(t_{j}^{+}, t_{k}\right) I_{i k}\left(t_{k}, y\left(t_{k}\right)\right) \\
& -\mu \sum_{t_{j} \leq t_{k}<t_{j}+\omega} G_{i}\left(t_{j}, t_{k}\right) I_{i k}\left(t_{k}, y\left(t_{k}\right)\right) \\
= & \mu I_{i k}\left(t_{k}, y\left(t_{k}\right)\right) .
\end{aligned}
$$

Hence $y(t)=\left(y_{1}(t), y_{2}(t), \ldots, y_{n}(t)\right)^{T} \in X$ is a positive $\omega$-periodic solution of (1). Thus we complete the proof of Lemma 6.

Lemma 7. Assume that $\left(H_{1}\right)-\left(H_{4}\right)$ hold. Then $T: E \rightarrow E$ is well defined.

Proof. From (9), it is easy to verify that $(T y)(t)$ is continuous in $\left(t_{k}, t_{k+1}\right),(T y)\left(t_{k}^{+}\right)$and $(T y)\left(t_{k}^{-}\right)$exist, and $(T y)\left(t_{k}^{-}\right)=$ $(T y)\left(t_{k}\right)$ for each $k \in Z_{+}$. Moreover, for any $y \in E$

$$
\begin{aligned}
& (T y)(t+\omega) \\
& =\lambda \int_{t+\omega}^{t+2 \omega} G(t+\omega, s) b(s, y(s)) F(s, u(s)) d s \\
& \quad+\mu \sum_{t+\omega \leq t_{k}<t+2 \omega} G\left(t+\omega, t_{k}\right) I_{k}\left(t_{k}, y\left(t_{k}\right)\right)
\end{aligned}
$$

$$
\begin{aligned}
= & \lambda \int_{t}^{t+\omega} G(t+\omega, v+\omega) b(v+\omega, y(v+\omega)) \\
& \times F(v+\omega, u(v+\omega)) d v \\
& +\mu \sum_{t \leq t_{k}<t+\omega} G\left(t, t_{k}\right) I_{k}\left(t_{k}, y\left(t_{k}\right)\right) \\
= & \lambda \int_{t}^{t+\omega} G(t, s) b(s, y(s)) F(s, u(s)) d s \\
& +\mu \sum_{t \leq t_{k}<t+\omega} G\left(t, t_{k}\right) I_{k}\left(t_{k}, y\left(t_{k}\right)\right) \\
= & (T y)(t) .
\end{aligned}
$$

Therefore, $(T y) \in X$. From (9), we have

$$
\begin{aligned}
\left|T_{i} y\right|_{0} \leq \beta_{i}\left[\lambda \int_{0}^{\omega}\left|b_{i}(s, y(s)) f_{i}(s, u(s))\right| d s\right. & \\
& \left.+\mu \sum_{t \leq t_{k}<t+\omega} I_{i k}\left(t_{k}, y\left(t_{k}\right)\right)\right] .
\end{aligned}
$$

On the other hand, we obtain

$$
\begin{aligned}
&\left(T_{i} y\right)(t) \geq \alpha_{i}\left[\lambda \int_{0}^{\omega}\left|b_{i}(s, y(s)) f_{i}(s, u(s))\right| d s\right.\left.+\mu \sum_{t \leq t_{k}<t+\omega} I_{i k}\left(t_{k}, y\left(t_{k}\right)\right)\right] \\
& \geq \frac{\alpha_{i}}{\beta_{i}}\left|T_{i} y\right|_{0} \\
& \geq \sigma\left|T_{i} y\right|_{0} .
\end{aligned}
$$

Therefore, $T y \in E$. This completes the proof of Lemma 7.

Lemma 8. Assume that $\left(H_{1}\right)-\left(H_{4}\right)$ hold. Then $T: E \rightarrow E$ is completely continuous.

Proof. We first show that $T$ is continuous. By $\left(H_{3}\right)-\left(H_{4}\right), f$ and $H_{k}$ are continuous in $y$; it follows that for any $\epsilon>0$, let $\delta>0$ be small enough to satisfy that if $y, y^{*} \in E$, with $\left|y-y^{*}\right|<\delta$,

$$
\begin{gathered}
\left|f(s, u(s))-f\left(s, u^{*}(s)\right)\right|<\frac{\epsilon}{2 \bar{B} \lambda \beta \omega}, \quad s \in R, \\
\left|H_{k}\left(t_{k}, y\left(t_{k}\right)\right)-I_{k}\left(t_{k}, y^{*}\left(t_{k}\right)\right)\right|<\frac{\epsilon}{2 \beta \mu q}, \quad k \in Z_{+} .
\end{gathered}
$$


Therefore,

$$
\begin{aligned}
& \left\|(T y)(t)-\left(T y^{*}\right)(t)\right\| \\
& =\sum_{i=1}^{n}\left|T_{i} y-T_{i} y^{*}\right|_{0} \\
& \leq \beta \sum_{i=1}^{n} \lambda \int_{t}^{t+\omega} \mid b_{i}(s, y(s)) f_{i}(s, u(s)) \\
& \quad-b_{i}(s, y(s)) f_{i}\left(s, u^{*}(s)\right) \mid d s \\
& +\beta \mu \sum_{i=1}^{n} \sum_{t \leq t_{k}<t+\omega} \mid I_{i k}\left(t_{k}, y\left(t_{k}\right)\right) \\
& \quad-h_{i k}\left(t_{k}, y^{*}\left(t_{k}\right)\right) \mid \\
& <\beta \lambda \bar{B} \omega \frac{\epsilon}{2 \bar{B} \lambda \beta \omega}+\beta \mu q \frac{\epsilon}{2 \beta \mu q} \\
& =\epsilon,
\end{aligned}
$$

which implies that $T$ is continuous on $E$.

Next we show that $T$ maps a bounded set into a bounded set. Indeed, let $C \in E$ be a bounded set. For any $t \in R$ and $x \in C$, by (9), we have

$$
\begin{gathered}
\|(T y)(t)\|=\sum_{i=1}^{n}\left|T_{i} y\right|_{0} \\
\leq \beta\left[\lambda \sum_{i=1}^{n} \int_{t}^{t+\omega}\left|b_{i}(s, y(s)) f_{i}(s, u(s))\right| d s\right. \\
\left.\quad+\mu \sum_{i=1}^{n} \sum_{t \leq t_{k}<t+\omega}\left|I_{i k}\left(t_{k}, y\left(t_{k}\right)\right)\right|\right] \\
=\beta\left[\int_{0}^{\omega} b_{2 i}(s) F(s, u(s)) d s\right. \\
\left.\quad+\sum_{t \leq t_{k}<t+\omega}\left|I_{k}\left(t_{k}, y\left(t_{k}\right)\right)\right|\right] .
\end{gathered}
$$

Since $C$ is bounded, in view of the continuity of $T$, it follows from (21) that $T y$ is bounded and $\{T y: y \in C\}$ is uniformly bounded. Finally, we show that the family of functions $\{T y$ : $y \in C\}$ is equicontinuous on $[0, \omega]$. Let $\theta_{1}, \theta_{2} \in[0, \omega]$ with $\theta_{1}<\theta_{2}$. From (9), for any $y \in C$, we have

$$
\begin{aligned}
& \left\|(T y)\left(\theta_{2}\right)-(T y)\left(\theta_{1}\right)\right\| \\
& \leq \lambda \sum_{i=1}^{n}\left[\int_{\theta_{1}}^{\theta_{2}}\left(G_{i}\left(\theta_{2}, s\right)-G_{i}\left(\theta_{1}, s\right)\right)\right. \\
& \quad \times b_{i}(s, y(s)) f_{i}(s, u(s)) d s \\
& \quad+\int_{\theta_{2}}^{\theta_{1}+\omega}\left(G_{i}\left(\theta_{2}, s\right)-G_{i}\left(\theta_{1}, s\right)\right) \\
& \quad \times b_{i}(s, y(s)) f_{i}(s, u(s)) d s
\end{aligned}
$$

$$
\begin{gathered}
+\sum_{\theta_{1} \leq t_{k}<\theta_{2}}\left(G_{i}\left(\theta_{2}, t_{k}\right)-G_{i}\left(\theta_{1}, t_{k}\right)\right) \\
\times\left|I_{i k}\left(t_{k}, y\left(t_{k}\right)\right)\right| \\
+\sum_{\theta_{2} \leq t_{k}<\theta_{1}+\omega}\left(G_{i}\left(\theta_{2}, t_{k}\right)-G_{i}\left(\theta_{1}, t_{k}\right)\right) \\
\left.\times\left|I_{i k}\left(t_{k}, y\left(t_{k}\right)\right)\right|\right] .
\end{gathered}
$$

Since for $y \in C, t \in[0, \omega], 0 \leq k \leq q, b_{i}(t, y(t)), f_{i}(t, u(t))$, and $I_{i k}\left(t_{k}, y\left(t_{k}\right)\right)$ are uniformly bounded in $X$, in view of (23), it is easy to see that when $\theta_{2}-\theta_{1}$ tends to zero, $\mid(T y)\left(\theta_{2}\right)-$ $(T y)\left(\theta_{1}\right) \mid$ tends uniformly to zero in $X$. Hence, $\{T y: y \in C\}$ is a family of uniformly bounded and equicontinuous functions on $[0, \omega]$. By Arzela-Ascoli theorem, the operator $T$ is completely continuous. The proof of Lemma 8 is complete.

Our main results of this paper are as follows.

Theorem 9. Assume that $\left(H_{1}\right)-\left(H_{4}\right)$ and

$\left(H_{5}\right)$ there exists a $R>0$ such that $F(t, u) \geq(R /$ $\left.\left(2 \alpha \sigma B^{\prime}(\xi) \lambda \omega\right)\right), \sum_{t \leq t_{k}<t+\omega} I_{k}\left(t_{k}, y\left(t_{k}\right)\right) \geq(R /(2 \alpha \sigma \mu))$, for $\|u\| \leq\|y\| \in[\sigma R, R]$;

$$
\left(H_{6}\right) F^{0}=I^{0}=F^{\infty}=I^{\infty}=0
$$

hold. Then, (1) has two positive $\omega$-periodic solutions.

Proof. First, we define $\Omega_{R}=\{y \in X:\|y\|<R\}$; then $\Omega_{R}$ is an open subset of $X$. Then, for any $y \in E \cap \partial \Omega_{r}$, we have $y(t) \geq \sigma R$. Consequently,

$$
\begin{gathered}
u_{j}(t)=y\left(c_{j}(t)\right) \geq \sigma\|y\| \geq \sigma\|u(t)\|, \\
u_{n}(t)=\int_{-\infty}^{t} k(t-\xi) y(\xi) d \xi \geq \sigma\|y\| \geq \sigma\|u(t)\| ;
\end{gathered}
$$

then

$$
\|u(t)\|=\max _{1 \leq j \leq n-1}\left\{y\left(c_{j}(t)\right), \int_{-\infty}^{t} k(t-\xi) y(\xi) d \xi\right\} \geq \sigma R,
$$

and from the definition of $u(t)$, we know $\|u(t)\| \leq\|y(t)\|$. From (9), $\left(H_{5}\right)$, and Lemma 5, we get

$$
\begin{aligned}
\|(T y)(t)\|= & \sum_{i=1}^{n}\left|T_{i} y\right|_{0} \\
=\sum_{i=1}^{n}\left[\lambda \int_{t}^{t+\omega}\left|G_{i}(t, s) b_{i}(s, y(s)) f_{i}(s, u(s))\right| d s\right. & \\
& \left.\quad+\mu \sum_{t \leq t_{k}<t+\omega}\left|G_{i}\left(t, t_{k}\right) I_{i k}\left(t_{k}, y\left(t_{k}\right)\right)\right|\right]
\end{aligned}
$$




$$
\begin{aligned}
& \geq \lambda B^{\prime}(\xi) \sum_{i=1}^{n} \int_{t}^{t+\omega}\left|G_{i}(t, s) f_{i}(s, u(s))\right| d s \\
& +\mu \sum_{i=1}^{n} \sum_{t \leq t_{k}<t+\omega}\left|G_{i}\left(t, t_{k}\right) I_{i k}\left(t_{k}, y\left(t_{k}\right)\right)\right| \\
& =\alpha\left[\lambda B^{\prime}(\xi) \int_{0}^{\omega}|F(s, u(s))| d s\right. \\
& \left.\quad+\mu \sum_{t \leq t_{k}<t+\omega}\left|I_{k}\left(t_{k}, y\left(t_{k}\right)\right)\right|\right] \\
& \geq \alpha \sigma\left(\lambda B^{\prime}(\xi) \omega \frac{R}{2 \alpha \sigma B^{\prime}(\xi) \lambda \omega}+\frac{R \mu}{2 \alpha \sigma \mu}\right)=R \\
& >\|y\| .
\end{aligned}
$$

This yields

$$
\|T y\|>\|y\|, \quad \text { for any } y \in E \cap \partial \Omega_{R} .
$$

On the other hand, if $F^{0}=I^{0}=0$ holds, then we can choose $0<R_{1}<R$, so that $\int_{0}^{\omega}|f(t, u(t))| d t \leq \epsilon\|u\|$; from the definition of $u(t)$, we know $\|u\| \leq\|y\|$. Thus, we have $\int_{0}^{\omega}|f(t, u(t))| d t \leq \epsilon\|y\|, \sum_{t \leq t_{k}<t+\omega}\left|I_{k}\left(t_{k}, y\left(t_{k}\right)\right)\right| \leq \epsilon\|y\|$ for $y \in\left[0, R_{1}\right], t \in[0, \omega], 1 \leq k<q$, where constant $\epsilon>0$ satisfies $\epsilon \beta(\lambda B(\xi)+\mu) \leq 1$. By (9) and Lemma 5 , we can obtain

$(T y)(t)$

$$
\begin{aligned}
= & \sum_{i=1}^{n}\left(T_{i} y\right) \\
= & \sum_{i=1}^{n}\left[\lambda \int_{t}^{t+\omega} G_{i}(t, s) b_{i}(s, y(s)) f_{i}(s, u(s)) d s\right. \\
& \left.+\mu \sum_{t \leq t_{k}<t+\omega} G_{i}\left(t, t_{k}\right) I_{i k}\left(t_{k}, y\left(t_{k}\right)\right)\right] \\
\leq & \lambda B(\xi) \sum_{i=1}^{n} \int_{t}^{t+\omega}\left|G_{i}(t, s) f_{i}(s, u(s))\right| d s \\
& +\mu \sum_{i=1}^{n} \sum_{t \leq t_{k}<t+\omega}\left|G_{i}\left(t, t_{k}\right) I_{i k}\left(t_{k}, y\left(t_{k}\right)\right)\right| \\
= & \beta\left[\lambda B(\xi) \int_{0}^{\omega}|F(s, u(s))| d s\right. \\
& \left.+\mu \sum_{t \leq t_{k}<t+\omega}\left|I_{k}\left(t_{k}, y\left(t_{k}\right)\right)\right|\right] \\
\leq & \epsilon \beta(\lambda B(\xi)+\mu)\|y\| \\
\leq & \|y\| .
\end{aligned}
$$

This yields

$$
\|(T y)(t)\| \leq\|y\|, \quad \text { for any } y \in E \cap \partial \Omega_{R_{1}} .
$$

In view of (27) and (29), by Lemma 5 , it follows that $T$ has a fixed point $y^{*} \in E \cap\left(\Omega_{R} \backslash \overline{\Omega_{R_{1}}}\right)$ with $R_{1}<\left\|y^{*}\right\|<R$, which is a positive $\omega$-periodic solution of (1).

Likewise, in view of $F^{\infty}=I^{\infty}=0$, for any $0<\epsilon<$ $(1 /(4 \beta B(\xi) \lambda \omega))$, there is $N>0$ such that

$$
F(t, u(t)) \leq \epsilon\|u\|, \quad \text { for }\|u\|>N .
$$

Let $\Omega_{R_{2}}=\left\{y \in X:\|y\|<R_{2}\right\}$, where

$$
\begin{aligned}
R_{2} \geq & 4 N+4 B(\xi) \lambda \omega \max _{t \in[0, \omega],\|u\| \leq N} F(t, u(t)) \\
& +2 \beta \mu \sum_{t \leq t_{k}<t+\omega}\left|I_{k}\left(t_{k}, y\left(t_{k}\right)\right)\right| .
\end{aligned}
$$

Then for any $y \in E \bigcap \partial \Omega_{R_{2}}$, from (9), (30), and (31), we have

$$
\begin{aligned}
\|(T y)(t)\|= & \sum_{i=1}^{n}\left|T_{i} y\right|_{0} \\
= & \sum_{i=1}^{n}\left[\lambda \int_{t}^{t+\omega}\left|G_{i}(t, s) b_{i}(s, y(s)) f_{i}(s, u(s))\right| d s\right. \\
& \left.+\mu \sum_{t \leq t_{k}<t+\omega}\left|G_{i}\left(t, t_{k}\right) I_{i k}\left(t_{k}, y\left(t_{k}\right)\right)\right|\right] \\
\leq & \beta B(\xi) \sum_{i=1}^{n} \int_{t}^{t+\omega}\left|G_{i}(t, s) f_{i}(s, u(s))\right| d s \\
& +\mu \sum_{i=1}^{n} \sum_{t \leq t_{k}<t+\omega}\left|G_{i}\left(t, t_{k}\right) I_{i k}\left(t_{k}, y\left(t_{k}\right)\right)\right| \\
= & {\left[\lambda B(\xi) \int_{t}^{t+\omega}|F(s, u(s))| d s\right.} \\
& \left.+\mu \sum_{t \leq t_{k}<t+\omega}\left|I_{k}\left(t_{k}, y\left(t_{k}\right)\right)\right|\right] \\
= & \|y\|, \quad \frac{R_{2}}{4}+\frac{\|y\|}{4}+\frac{R_{2}}{2} \\
& +\mu(\xi)\left[\int_{I_{1}}|F(s, u(s))| d s\right. \\
& \left.+\int_{I_{2}}|F(s, u(s))| d s\right] \\
&
\end{aligned}
$$


where $I_{1}=\{s \in[0, \omega],\|u(s)\| \leq N\}, I_{2}=\{s \in[0, \omega],\|u(s)\|>$ $N\}$. This yields

$$
\|T y\|<\|y\|, \quad \text { for any } y \in E \cap \partial \Omega_{R_{2}} .
$$

In view of (27) and (33), by Lemma 5 , it follows that $T$ has a fixed point $\bar{y} \in E \cap\left(\Omega_{R_{2}} \backslash \overline{\Omega_{R}}\right)$ with $R<\|\bar{y}\|<R_{2}$, which is a positive $\omega$-periodic solution of (1). Therefore (1) has at least two positive periodic solutions; that is, $R_{1}<\left\|y^{*}\right\|<R<$ $\|\bar{y}\|<R_{2}$. This proves Theorem 9 .

Corollary 10. Assume that $\left(H_{1}\right)-\left(H_{4}\right)$ and

$\left(H_{5}\right)$ There exists a $R>0$ such that $F(t, u) \geq(R /$ $\left.2 \alpha \sigma B^{\prime}(\xi) \lambda \omega\right), \sum_{t \leq t_{k}<t+\omega} I_{k}\left(t_{k}, y\left(t_{k}\right)\right) \geq(R / 2 \alpha \sigma \mu)$, for $\|u\| \leq\|y\| \in[\sigma R, R]$

$\left(H_{7}\right) F^{0}=I^{0}=0$, or $F^{\infty}=I^{\infty}=0$

hold. Then, (1) has a positive $\omega$-periodic solution.

Theorem 11. Assume that $\left(H_{1}\right)-\left(H_{4}\right)$ and

$\left(H_{8}\right)$ there exists a $r>0$ such that $F(t, u) \leq(r / 2 \beta B(\xi) \lambda \omega)$, $\sum_{t \leq t_{k}<t+\omega} I_{k}\left(t_{k}, y\left(t_{k}\right)\right) \leq(r / 2 \beta \mu)$, for $\|u\| \leq\|y\| \leq r$;

$\left(H_{9}\right) F_{0}=I_{0}=F_{\infty}=I_{\infty}=\infty$

hold. Then, (1) has two positive $\omega$-periodic solutions.

Proof. We define $\Omega_{r}=\{y \in X:\|y\|<r\}$, where R satisfied $\left(H_{8}\right)$; then $\Omega_{r}$ is an open subset of $X$ and $0 \in \Omega_{r}$. For any $y \in$ $E \cap \partial \Omega_{r}$, by the definition of $u(t)$, we get $\|u(t)\| \leq\|y(t)\|=r$. Furthermore, by $(9),\left(H_{8}\right)$, and Lemma 5 , we have

$$
\begin{aligned}
\|(T y)(t)\|= & \sum_{i=1}^{n}\left|T_{i} y\right|_{0} \\
= & \sum_{i=1}^{n}\left[\lambda \int_{t}^{t+\omega}\left|G_{i}(t, s) b_{i}(s, y(s)) f_{i}(s, u(s))\right|\right. \\
& \left.+\mu \sum_{t \leq t_{k}<t+\omega}\left|G_{i}\left(t, t_{k}\right) I_{i k}\left(t_{k}, y\left(t_{k}\right)\right)\right|\right] \\
\leq & \lambda B(\xi) \sum_{i=1}^{n} \int_{t}^{t+\omega}\left|G_{i}(t, s) f_{i}(s, u(s))\right| d s \\
& +\mu \sum_{i=1}^{n} \sum_{t \leq t_{k}<t+\omega}\left|G_{i}\left(t, t_{k}\right) I_{i k}\left(t_{k}, y\left(t_{k}\right)\right)\right| \\
= & \beta\left[\lambda B(\xi) \int_{0}^{\omega}|F(s, u(s))| d s\right. \\
& \left.\quad+\mu \sum_{t \leq t_{k}<t+\omega}\left|I_{k}\left(t_{k}, y\left(t_{k}\right)\right)\right|\right] \\
\leq & \beta\left(\lambda B(\xi) \omega \frac{r}{2 \beta B(\xi) \lambda \omega}+\frac{r}{2 \beta \mu}\right) \leq r=\|y\| .
\end{aligned}
$$

This implies for any $y \in E \cap \partial \Omega_{r}$

$$
\|T y\| \leq\|y\| \text {. }
$$

On the one hand, since $F_{0}=I_{0}=\infty$, then for any $M_{1}>$ $1 /\left(2 \alpha B^{\prime}(\xi) \sigma \lambda \omega\right)$ there exists $0<r_{1}<r$ such that

$$
\begin{gathered}
F(t, u(t)) \geq M_{1}\|u\|, \quad \text { for }\|u\|<r_{1}, \\
u_{j} \geq \sigma\|u\|, \quad j=1,2, \ldots, n .
\end{gathered}
$$

Letting $\Omega_{r_{1}}=\left\{y \in X:\|y\|<r_{1}\right\}$, then for any $y \in$ $E \bigcap \partial \Omega_{r_{1}}$, one has $\|y(t)\|=r_{1}, \sum_{t \leq t_{k}<t+\omega}\left|H_{k}\left(t_{k}, y\left(t_{k}\right)\right)\right| \geq$ $(1 /(2 \alpha \mu))\|y(t)\|=\left(r_{1} /(2 \alpha \mu)\right), y(t) \geq \sigma\|y(t)\|=\sigma r_{1}$. Consequently,

$$
\begin{gathered}
u_{j}(t)=y\left(c_{j}(t)\right) \geq \sigma\|y\| \geq \sigma\|u(t)\|, \\
u_{n}(t)=\int_{-\infty}^{t} k(t-\xi) y(\xi) d \xi \geq \sigma\|y\| \geq \sigma\|u(t)\|, \\
\sigma r_{1} \leq\|u(t)\| \\
=\max _{1 \leq j \leq n-1}\left\{y\left(c_{j}(t)\right), \int_{-\infty}^{t} k(t-\xi) y(\xi) d \xi\right\} \\
\leq\|y(t)\|=r_{1},
\end{gathered}
$$

where, together with (42) and Lemma 5, we have

$$
\begin{aligned}
\|(T y)(t)\|= & \sum_{i=1}^{n}\left|T_{i} y\right|_{0} \\
= & \sum_{i=1}^{n}\left[\lambda \int_{t}^{t+\omega}\left|G_{i}(t, s) b_{i}(s, y(s)) f_{i}(s, u(s))\right| d s\right. \\
& \left.\quad+\mu \sum_{t \leq t_{k}<t+\omega}\left|G_{i}\left(t, t_{k}\right) I_{i k}\left(t_{k}, y\left(t_{k}\right)\right)\right|\right] \\
\geq \alpha B^{\prime}(\xi) \sum_{i=1}^{n} \int_{t}^{t+\omega}\left|G_{i}(t, s) f_{i}(s, u(s))\right| d s & \\
& +\mu \sum_{i=1}^{n} \sum_{t \leq t_{k}<t+\omega}\left|G_{i}\left(t, t_{k}\right) I_{i k}\left(t_{k}, y\left(t_{k}\right)\right)\right| \\
= & \alpha\left[\lambda B^{\prime}(\xi) \int_{t}^{t+\omega}|F(s, u(s))| d s\right. \\
& \left.\quad+\mu \sum_{t \leq t_{k}<t+\omega}\left|I_{k}\left(t_{k}, y\left(t_{k}\right)\right)\right|\right] \\
\geq & \alpha \lambda B^{\prime}(\xi) M_{1} \sigma\|y(t)\| \omega+\frac{\|y(t)\|}{2} \\
= & \|y\| .
\end{aligned}
$$

This implies for any $y \in E \cap \partial \Omega_{r_{1}}$

$$
\|T y\| \geq\|y\| \text {. }
$$


In view of (35) and (39), by Lemma 2, it follows that $T$ has a fixed point $y^{*} \in E \cap\left(\Omega_{R} \backslash \overline{\Omega_{r_{1}}}\right)$ with $r_{1}<\left\|y^{*}\right\|<r$, which is a positive $\omega$-periodic solution of (1). On the other hand, if $F_{\infty}=I_{\infty}=\infty$, then for any $M_{2}>\left(1 / 2 \alpha B^{\prime}(\xi) \sigma \lambda \omega\right)$ there exists $r_{2}>r$ such that

$$
\begin{gathered}
F(t, u(t)) \geq M_{2}\|u\|, \quad \text { for }\|u\|>r_{2}, \\
u_{j} \geq \sigma\|u\|, \quad j=1,2, \ldots, n .
\end{gathered}
$$

Letting $\Omega_{r_{2}}=\left\{y \in X:\|y\|<r_{2}\right\}$, then for any $y \in$ $E \cap \partial \Omega_{r_{2}}$, one has $\|y(t)\|=r_{2}, \sum_{t \leq t_{k}<t+\omega}\left|I_{k}\left(t_{k}, y\left(t_{k}\right)\right)\right| \geq$ $(1 / 2 \alpha \mu)\|y(t)\|=\left(r_{2} / 2 \alpha \mu\right), y(t) \geq \sigma\|y(t)\|=\sigma r_{2}$. Consequently,

$$
\begin{gathered}
u_{j}(t)=y\left(c_{j}(t)\right) \geq \sigma\|y\| \geq \sigma\|u(t)\|, \\
u_{n}(t)=\int_{-\infty}^{t} k(t-\xi) y(\xi) d \xi \geq \sigma\|y\| \geq \sigma\|u(t)\|, \\
\|u(t)\|=\max _{1 \leq j \leq n-1}\left\{y\left(c_{j}(t)\right), \int_{-\infty}^{t} k(t-\xi) y(\xi) d \xi\right\} \\
\geq \sigma\|y(t)\|=\sigma r_{2},
\end{gathered}
$$

where, together with (41) and Lemma 5, we have

$$
\begin{aligned}
& \|(T y)(t)\|=\sum_{i=1}^{n}\left|T_{i} y\right|_{0} \\
& =\sum_{i=1}^{n}\left[\lambda \int_{t}^{t+\omega}\left|G_{i}(t, s) b_{i}(s, y(s)) f_{i}(s, u(s))\right| d s\right. \\
& \left.+\mu \sum_{t \leq t_{k}<t+\omega}\left|G_{i}\left(t, t_{k}\right) I_{i k}\left(t_{k}, y\left(t_{k}\right)\right)\right|\right] \\
& \geq \alpha B^{\prime}(\xi) \sum_{i=1}^{n} \int_{t}^{t+\omega}\left|G_{i}(t, s) f_{i}(s, u(s))\right| d s \\
& +\mu \sum_{i=1}^{n} \sum_{t \leq t_{k}<t+\omega}\left|G_{i}\left(t, t_{k}\right) I_{i k}\left(t_{k}, y\left(t_{k}\right)\right)\right| \\
& =\alpha\left[\lambda B^{\prime}(\xi) \int_{t}^{t+\omega}|F(s, u(s))| d s\right. \\
& \left.+\mu \sum_{t \leq t_{k}<t+\omega}\left|I_{k}\left(t_{k}, y\left(t_{k}\right)\right)\right|\right] \\
& \geq \alpha \lambda B^{\prime}(\xi) M_{2} \sigma r_{2} \omega+\frac{\|y(t)\|}{2} \\
& \geq\|y\| \text {. }
\end{aligned}
$$

This yields

$$
\|(T y)(t)\| \geq\|y\|, \quad \text { for any } y \in E \cap \partial \Omega_{r_{2}} .
$$

In view of (35) and (43), by Lemma 2 , it follows that $T$ has a fixed point $\bar{y} \in E \cap\left(\Omega_{r_{2}} \backslash \overline{\Omega_{r}}\right)$ with $r<\|\bar{y}\|<r_{2}$, which is a positive $\omega$-periodic solution of (1). Therefore, (1) has at least two positive periodic solutions; that is $r_{1}<\left\|y^{*}\right\|<r<\|\bar{y}\|<$ $r_{2}$. This proves Theorem 11 .

Corollary 12. Assume that $\left(H_{1}\right)-\left(H_{4}\right)$ and

$\left(H_{8}\right)$ there exists a $r>0$ such that $F(t, u) \leq(r / 2 \beta B(\xi) \lambda \omega)$, $\sum_{t \leq t_{k}<t+\omega} H_{k}\left(t_{k}, y\left(t_{k}\right)\right) \leq(r / 2 \beta \mu)$, for $\|u\| \leq\|y\| \leq r ;$

$\left(H_{10}\right) F_{0}=I_{0}=\infty$, or $F_{\infty}=I_{\infty}=\infty$;

hold. Then, (1) has a positive $\omega$-periodic solution.

Theorem 13. Assume that $\left(H_{1}\right)-\left(H_{5}\right)$, and $\left(H_{8}\right)$ hold. Then, (1) has a positive $\omega$-periodic solution $y$ with $\|y\|$ lying between $R$ and $r$, which are defined in $\left(H_{5}\right)$ and $\left(H_{8}\right)$, respectively.

Proof. Without loss of generality, we may assume that $r<R$; then for any $y \in E \cap \partial \Omega_{r}$, by the definition of $u(t)$, we get $\|u(t)\| \leq\|y(t)\|=r$. Furthermore, by $(9),\left(H_{8}\right)$, and Lemma 5, we have

$$
\begin{aligned}
\|(T y)(t)\|= & \sum_{i=1}^{n}\left|T_{i} y\right|_{0} \\
= & \sum_{i=1}^{n}\left[\lambda \int_{t}^{t+\omega}\left|G_{i}(t, s) b_{i}(s, y(s)) f_{i}(s, u(s))\right|\right. \\
& \left.+\mu \sum_{t \leq t_{k}<t+\omega}\left|G_{i}\left(t, t_{k}\right) I_{i k}\left(t_{k}, y\left(t_{k}\right)\right)\right|\right] \\
\leq & \lambda B(\xi) \sum_{i=1}^{n} \int_{t}^{t+\omega}\left|G_{i}(t, s) f_{i}(s, u(s))\right| d s \\
& +\mu \sum_{i=1}^{n} \sum_{t \leq t_{k}<t+\omega}\left|G_{i}\left(t, t_{k}\right) I_{i k}\left(t_{k}, y\left(t_{k}\right)\right)\right| \\
= & {\left[\lambda B(\xi) \int_{0}^{\omega}|F(s, u(s))| d s\right.} \\
\leq & +\mu\left(\lambda B(\xi) \omega \frac{r}{2 \beta B(\xi) \lambda \omega}+\frac{r}{2 \beta \mu}\right) \\
\leq & \left.\quad\|y\| . I_{k}\left(t_{k}, y\left(t_{k}\right)\right) \mid\right] \\
& \\
& \\
& \\
& \\
& \\
&
\end{aligned}
$$

This implies for any $y \in E \cap \partial \Omega_{r}$

$$
\|T y\| \leq\|y\|
$$


Now, we let $\Omega_{R}=\{y \in X:\|y\|<R\}$; then $\Omega_{R}$ is an open subset of $X$. Then, for any $y \in E \cap \partial \Omega_{R}$, we have $y(t) \geq \sigma R$. Consequently,

$$
\begin{gathered}
u_{j}(t)=y\left(c_{j}(t)\right) \geq \sigma\|y\| \geq \sigma\|u(t)\|, \\
u_{n}(t)=\int_{-\infty}^{t} k(t-\xi) y(\xi) d \xi \geq \sigma\|y\| \geq \sigma\|u(t)\|, \\
\|u(t)\|=\max _{1 \leq j \leq n-1}\left\{y\left(c_{j}(t)\right), \int_{-\infty}^{t} k(t-\xi) y(\xi) d \xi\right\} \\
\geq \sigma R,
\end{gathered}
$$

and from the definition of $u(t)$, we know $\|u(t)\| \leq\|y(t)\|$. From (9), $\left(H_{5}\right)$, and Lemma 5, we get

$$
\begin{aligned}
\|(T y)(t)\|= & \sum_{i=1}^{n}\left|T_{i} y\right|_{0} \\
= & \sum_{i=1}^{n}\left[\lambda \int_{t}^{t+\omega}\left|G_{i}(t, s) b_{i}(s, y(s)) f_{i}(s, u(s))\right| d s\right. \\
& \left.\quad+\mu \sum_{t \leq t_{k}<t+\omega}\left|G_{i}\left(t, t_{k}\right) I_{i k}\left(t_{k}, y\left(t_{k}\right)\right)\right|\right] \\
\geq & \lambda B^{\prime}(\xi) \sum_{i=1}^{n} \int_{t}^{t+\omega}\left|G_{i}(t, s) f_{i}(s, u(s))\right| d s \\
& +\mu \sum_{i=1}^{n} \sum_{t \leq t_{k}<t+\omega}\left|G_{i}\left(t, t_{k}\right) I_{i k}\left(t_{k}, y\left(t_{k}\right)\right)\right| \\
= & \alpha\left[B^{\prime}(\xi) \int_{0}^{\omega}|F(s, u(s))| d s\right. \\
& \left.\quad+\mu \sum_{t \leq t_{k}<t+\omega}\left|I_{k}\left(t_{k}, y\left(t_{k}\right)\right)\right|\right] \\
\geq & \alpha \sigma\left(\lambda B^{\prime}(\xi) \omega \frac{R}{2 \alpha \sigma B^{\prime}(\xi) \lambda \omega}+\frac{R \mu}{2 \alpha \sigma \mu}\right)=R \\
> & \|y\| .
\end{aligned}
$$

This yields

$$
\|T y\|>\|y\|, \quad \text { for any } y \in E \cap \partial \Omega_{R} .
$$

In view of (45) and (48), by Lemma 2 , it follows that $T$ has a fixed point $y^{*} \in E \cap\left(\Omega_{R} \backslash \overline{\Omega_{r}}\right)$ with $r<\left\|y^{*}\right\|<R$, which is a positive $\omega$-periodic solution of (1). This proves Theorem 13 .

Theorem 14. In addition to $\left(H_{1}\right)-\left(H_{4}\right)$, suppose the following conditions hold:

$$
\begin{aligned}
& \left(H_{11}\right) 0<F^{0} \leq(1 / 2 \beta \lambda B(\xi) \omega), 0<I^{0} \leq(1 / 2 \beta \mu) ; \\
& \left(H_{12}\right) F_{\infty} \geq\left(1 / 2 \alpha \sigma \lambda B^{\prime}(\xi) \omega\right), I_{\infty} \geq(1 / 2 \alpha \sigma \mu) ;
\end{aligned}
$$

then, (1) has a positive $\omega$-periodic solution.
Proof. In view of $0<F^{0} \leq(1 / 2 \beta \lambda B(\xi) \omega), 0<I^{0} \leq 1 /(2 \beta \mu)$, there exists a sufficiently small $r>0$ such that

$$
\begin{gathered}
\frac{\int_{0}^{\omega}|F(t, u(t))| d t}{\|u\|} \leq \frac{1}{2 \beta \lambda B(\xi) \omega}, \quad \text { for any }\|u(t)\| \leq r, \\
\frac{\sum_{t \leq t_{k}<t+\omega}\left|I_{k}(t, y)\right|}{\|y\|} \leq \frac{1}{2 \beta \mu}, \quad \text { for any }\|y(t)\| \leq r,
\end{gathered}
$$

which yields

$$
\begin{aligned}
\int_{0}^{\omega}|F(t, u(t))| d t & \leq \frac{1}{2 \beta \lambda B(\xi) \omega}\|u\| \\
& \leq \frac{r}{2 \beta \lambda B(\xi) \omega}, \quad \text { for any }\|u(t)\| \leq r, \\
\sum_{t \leq t_{k}<t+\omega}\left|I_{k}(t, y)\right| & \leq \frac{1}{2 \beta \mu}\|y\| \\
& \leq \frac{r}{2 \beta \mu}, \quad \text { for any }\|y(t)\| \leq r .
\end{aligned}
$$

Therefore, condition $\left(H_{8}\right)$ is satisfied. On the other hand, since $F_{\infty} \geq\left(1 / 2 \alpha \sigma \lambda B^{\prime}(\xi) \omega\right), I_{\infty} \geq(1 / 2 \alpha \sigma \mu)$, there exists a sufficiently large $R>0(R>r)$ such that

$$
\begin{aligned}
& \frac{\int_{0}^{\omega}|F(t, u(t))| d t}{\|u\|} \\
& \geq \frac{1}{2 \alpha \sigma \lambda B^{\prime}(\xi) \omega}, \quad \text { for any }\|u(t)\| \in[\sigma R, R], \\
& \frac{\sum_{t \leq t_{k}<t+\omega}\left|I_{k}(t, y)\right|}{\|y\|} \\
& \quad \geq \frac{1}{2 \alpha \sigma \mu}, \quad \text { for any }\|y(t)\| \in[\sigma R, R],
\end{aligned}
$$

which yields

$$
\begin{aligned}
& \int_{0}^{\omega}|F(t, u(t))| d t \\
& \geq \frac{1}{2 \alpha \sigma \lambda B^{\prime}(\xi) \omega}\|u\| \\
& \geq \frac{R}{2 \alpha \sigma \lambda B^{\prime}(\xi) \omega}, \quad \text { for any }\|u(t)\| \in[\sigma R, R], \\
& \sum_{t \leq t_{k}<t+\omega}\left|I_{k}(t, y)\right| \geq \frac{1}{2 \alpha \sigma \mu}\|y\| \\
& \geq \frac{R}{2 \alpha \sigma \mu}, \quad \text { for any }\|y(t)\| \in[\sigma R, R] .
\end{aligned}
$$

Thus, condition $\left(H_{5}\right)$ is satisfied. By Theorem 13, we complete the proof. 
Theorem 15. Assume that $\left(H_{1}\right)-\left(H_{4}\right)$ and

$$
\begin{aligned}
& \left(H_{13}\right) F_{0}>\left(1 / 2 \alpha \sigma \lambda B^{\prime}(\xi) \omega\right), I_{0}>1 / 2 \alpha \sigma \mu ; \\
& \left(H_{14}\right) 0<F^{\infty} \leq(1 / 2 \beta \lambda B(\xi) \omega), 0<I^{\infty} \leq(1 /(2 \beta \sigma \mu))
\end{aligned}
$$

hold. Then, (1) has a positive $\omega$-periodic solution.

Proof. In view of $F_{0}>\left(1 / 2 \alpha \sigma \lambda B^{\prime}(\xi) \omega\right), I_{0}>(1 / 2 \alpha \sigma \mu)$, for $\epsilon=\min \left\{F_{0}-\left(1 / 2 \alpha \sigma \lambda B^{\prime}(\xi) \omega\right), I_{0}-(1 / 2 \alpha \sigma \mu)\right\}$, there exists a sufficiently small $r>0$ such that

$$
\begin{array}{r}
\frac{\int_{0}^{\omega}|F(t, u(t))| d t}{\|u\|} \geq F_{0}-\epsilon \geq \frac{1}{2 \alpha \sigma \lambda B^{\prime}(\xi) \omega}, \\
\text { for any } 0 \leq\|u(t)\| \leq r, \\
u_{j}(t) \geq \sigma\|u(t)\|, \\
\frac{\sum_{t \leq t_{k}<t+\omega}\left|I_{k}(t, y)\right|}{\|y\|} \geq I_{0}-\epsilon \geq \frac{1}{2 \alpha \sigma \mu}, \\
\text { for any }\|y(t)\| \in[\sigma r, r],
\end{array}
$$

which yields

$$
\begin{aligned}
& \int_{0}^{\omega}|F(t, u(t))| d t \\
& \quad \geq \frac{1}{2 \alpha \sigma \lambda B^{\prime}(\xi) \omega}\|u\| \\
& \geq \frac{r}{2 \alpha \sigma \lambda B^{\prime}(\xi) \omega}, \quad \text { for any }\|u(t)\| \in[\sigma r, r] \\
& \sum_{t \leq t_{k}<t+\omega}\left|I_{k}(t, y)\right| \geq \frac{1}{2 \alpha \sigma \mu}\|y\| \\
& \geq \frac{r}{2 \alpha \sigma \mu}, \text { for any }\|y(t)\| \in[\sigma r, r] .
\end{aligned}
$$

Therefore, condition $\left(\mathrm{H}_{5}\right)$ is satisfied. On the other hand, since $0<F^{\infty} \leq(1 / 2 \beta \lambda B(\xi) \omega), 0<I^{\infty} \leq(1 / 2 \beta \sigma \mu)$, for $\epsilon=\max \left\{(1 / 2 \beta \lambda B(\xi) \omega)-F^{\infty},(1 / 2 \beta \sigma \mu)-I^{\infty}\right\}$, there exists a sufficiently large $R(R>r)$ such that

$$
\begin{gathered}
\frac{\int_{0}^{\omega}|F(t, u(t))| d t}{\|u\|} \leq F^{\infty}+\epsilon \leq \frac{1}{2 \beta \lambda B(\xi) \omega}, \\
\text { for any }\|u(t)\| \geq R, \\
\frac{\sum_{t \leq t_{k}<t+\omega}\left|I_{k}(t, y)\right|}{\|y\| \quad} \leq I^{\infty}+\epsilon \leq \frac{1}{2 \beta \mu}, \\
\text { for any }\|y(t)\| \geq R .
\end{gathered}
$$

In the following, we consider two cases to prove $\left(H_{8}\right)$ to be satisfied: $\int_{0}^{\omega}|F(t, u(t))| d t, \sum_{t \leq t_{k}<t+\omega}\left|I_{k}(t, y)\right|$ are bounded or unbounded. The bounded case is clear. If $\int_{0}^{\omega}|F(t, u(t))| d t$, and $\sum_{t \leq t_{k}<t+\omega}\left|I_{k}(t, y)\right|$ are unbounded, then there exist $u^{*}, y^{*} \in R_{+}^{n}, R_{1}=\left\|y^{*}\right\| \geq\left\|u^{*}\right\| \geq R$ and $t_{0} \in[0, \omega]$ such that

$$
\begin{array}{r}
\int_{0}^{\omega}|F(t, u(t))| d t \leq \int_{0}^{\omega}\left|F\left(t, u^{*}(t)\right)\right| d t, \\
\text { for any }\|u(t)\| \leq\left\|u^{*}\right\| \leq R_{1}, \\
\sum_{t \leq t_{k}<t+\omega}\left|I_{k}(t, y)\right| \leq \sum_{t \leq t_{k}<t+\omega}\left|I_{k}\left(t, y^{*}\right)\right|, \\
\text { for any }\|y(t)\| \leq\left\|y^{*}\right\|+R_{1} .
\end{array}
$$

Since $R_{1}=\left\|y^{*}\right\| \geq\left\|u^{*}\right\| \geq R$, then we get

$$
\begin{aligned}
& \int_{0}^{\omega}|F(t, u(t))| d t \\
& \quad \leq \int_{0}^{\omega}\left|F\left(t, u^{*}(t)\right)\right| d t \leq \frac{\left\|u^{*}\right\|}{2 \beta \lambda B(\xi) \omega} \\
& \quad \leq \frac{\left\|y^{*}\right\|}{2 \beta \lambda B(\xi) \omega}=\frac{R_{1}}{2 \beta \lambda B(\xi) \omega},
\end{aligned}
$$$$
\text { for any }\|u(t)\| \leq\left\|u^{*}\right\| \leq R_{1},
$$

$$
\begin{aligned}
& \sum_{t \leq t_{k}<t+\omega}\left|I_{k}(t, y)\right| \\
& \leq \sum_{t \leq t_{k}<t+\omega}\left|I_{k}\left(t, y^{*}\right)\right| \leq \frac{\left\|y^{*}\right\|}{2 \beta \mu}=\frac{R_{1}}{2 \beta \mu}, \\
& \quad \text { for any }\|y(t)\| \leq\left\|y^{*}\right\|+R_{1} .
\end{aligned}
$$

Thus, condition $\left(H_{8}\right)$ is satisfied. By Theorem 14 , we complete the proof.

Theorem 16. Assume that $\left(H_{1}\right)-\left(H_{5}\right),\left(H_{11}\right)$, and $\left(H_{14}\right)$ hold. Then, (1) has at least two positive $\omega$-periodic solutions $y^{*}, \bar{y}$ satisfying $0<\left\|y^{*}\right\|<R<\|\bar{y}\|$, where $R$ is defined in $\left(H_{5}\right)$.

Proof. From $\left(\mathrm{H}_{5}\right)$ and the proof of Theorem 9, we know that there exists a sufficiently large $R>0$ such that

$$
\|T y\|>\|y\|, \quad \text { for any } y \in E \cap \partial \Omega_{R} .
$$

On the one hand, from $\left(H_{11}\right)$ and the proof of Theorem 14, we know that there exists a sufficiently small $r>0(r<R)$ such that

$$
\|T y\|<\|y\|, \quad \text { for any } y \in E \cap \partial \Omega_{r} .
$$

In view of (58) and (59), by Lemma 2, it follows that $T$ has a fixed point $y^{*} \in E \cap\left(\Omega_{R} \backslash \overline{\Omega_{r}}\right)$ with $r<\left\|y^{*}\right\|<R$, which is a positive $\omega$-periodic solution of (1).

On the other hand, from $\left(H_{14}\right)$ and the proof of Theorem 15 , we know that there exists a sufficiently large $R_{1}>$ $0\left(R_{1}>R\right)$ such that

$$
\|T y\|<\|y\|, \quad \text { for any } y \in E \cap \partial \Omega_{R_{1}} .
$$

In view of (58) and (60), by Lemma 2 , it follows that $T$ has a fixed point $\bar{y} \in E \cap\left(\Omega_{R_{1}} \backslash \overline{\Omega_{R}}\right)$ with $R<\|\bar{y}\|<R_{1}$, which is 
a positive $\omega$-periodic solution of (1). Thus, (1) has at least two positive $\omega$-periodic solutions $y^{*}, \bar{y}$ satisfying $r<\left\|y^{*}\right\|<R<$ $\|\bar{y}\|<R_{1}$. The proof is completed.

Corollary 17. In addition to $\left(H_{1}\right)-\left(H_{5}\right)$, suppose $\left(H_{11}\right)$ or $\left(H_{14}\right)$ holds. Then, (1) has at least one positive w-periodic solution.

Theorem 18. Assume that $\left(H_{1}\right)-\left(H_{4}\right),\left(H_{8}\right),\left(H_{12}\right)$, and $\left(H_{13}\right)$ hold. Then, (1) has at least two positive $\omega$-periodic solutions $y^{*}$, $\bar{y}$ satisfying $0<\left\|y^{*}\right\|<r<\|\bar{y}\|$, where $r$ is defined in $\left(H_{8}\right)$.

Proof. From $\left(H_{8}\right)$ and the proof of Theorem 9, we know that there exists a sufficiently small $r>0$ such that

$$
\|T y\|<\|y\|, \quad \text { for any } y \in E \cap \partial \Omega_{r} .
$$

On the one hand, from $\left(H_{12}\right)$ and the proof of Theorem 14, we know that there exists a sufficiently large $r_{1}>0\left(r_{1}>r\right)$ such that

$$
\|T y\|>\|y\|, \quad \text { for any } y \in E \cap \partial \Omega_{r_{1}} .
$$

In view of (61) and (62), by Lemma 2 , it follows that $T$ has a fixed point $y^{*} \in E \cap\left(\Omega_{r_{1}} \backslash \overline{\Omega_{r}}\right)$ with $r<\left\|y^{*}\right\|<r_{1}$, which is a positive $\omega$-periodic solution of (1).

On the other hand, from $\left(H_{13}\right)$ and the proof of Theorem 15, we know that there exists a sufficiently small $r^{*}>0\left(r^{*}<r\right)$ such that

$$
\|T y\|>\|y\|, \quad \text { for any } y \in E \cap \partial \Omega_{r}^{*} .
$$

In view of (61) and (63), by Lemma 2 , it follows that $T$ has a fixed point $y^{*} \in E \cap\left(\Omega_{r} \backslash \overline{\Omega_{r^{*}}}\right)$ with $r^{*}<\left\|y^{*}\right\|<r$, which is a positive $\omega$-periodic solution of (1). Thus, (1) has at least two positive $\omega$-periodic solutions $y^{*}, \bar{y}$ satisfying $r^{*}<\left\|y^{*}\right\|<r<$ $\|\bar{y}\|<r_{1}$. The proof is completed.

Corollary 19. In addition to $\left(H_{1}\right)-\left(H_{4}\right)$, and $\left(H_{8}\right)$, suppose $\left(H_{12}\right)$ or $\left(H_{13}\right)$ holds. Then, (1) has at least one positive $\omega$ periodic solution.

Theorem 20. Assume that $\left(H_{1}\right)-\left(H_{4}\right),\left(H_{6}\right),\left(H_{12}\right)$ hold. Then, (1) has at least two positive $\omega$-periodic solutions $y^{*}, \bar{y}$ satisfying $0<\left\|y^{*}\right\|<\|\bar{y}\|$.

Proof. From $\left(\mathrm{H}_{6}\right)$ and the proof of Theorem 9, if $F^{0}=I^{0}=0$, we know that there exists a sufficiently small $r>0$ such that

$$
\|T y\|<\|y\|, \quad \text { for any } y \in E \cap \partial \Omega_{r} .
$$

In view of $F^{\infty}=I^{\infty}=0$, we know that there exists a sufficiently large $R>0(R>r)$ such that

$$
\|T y\|<\|y\|, \quad \text { for any } y \in E \cap \partial \Omega_{R} .
$$

On the one hand, from $\left(H_{12}\right)$ and the proof of Theorem 14, we know that there exists a sufficiently large $r_{1}>0\left(R>r_{1}>r\right)$ such that

$$
\|T y\|>\|y\|, \quad \text { for any } y \in E \cap \partial \Omega_{r_{1}} .
$$

In view of (64) and (66), by Lemma 2, it follows that $T$ has a fixed point $y^{*} \in E \cap\left(\Omega_{r_{1}} \backslash \overline{\Omega_{r}}\right)$ with $r<\left\|y^{*}\right\|<r_{1}$, which is a positive $\omega$-periodic solution of (1). In view of (65) and (66), by Lemma 2 , it follows that $T$ has a fixed point $\bar{y} \in E \cap$ $\left(\Omega_{R} \backslash \overline{\Omega_{r_{1}}}\right)$ with $r_{1}<\|\bar{y}\|<R$, which is a positive $\omega$-periodic solution of (1). Thus, (1) has at least two positive $\omega$-periodic solutions $y^{*}, \bar{y}$ satisfying $r<\left\|y^{*}\right\|<r_{1}<\|\bar{y}\|<R$. The proof is completed.

Corollary 21. Assume that $\left(H_{1}\right)-\left(H_{4}\right),\left(H_{7}\right)$, and $\left(H_{12}\right)$ hold. Then, (1) has at least one positive $\omega$-periodic solution.

Theorem 22. Assume that $\left(H_{1}\right)-\left(H_{4}\right),\left(H_{6}\right)$, and $\left(H_{13}\right)$ hold. Then, (1) has at least two positive $\omega$-periodic solutions $y^{*}, \bar{y}$ satisfying $0<\left\|y^{*}\right\|<\|\bar{y}\|$.

Proof. From $\left(H_{13}\right)$ and the proof of Theorem 15, we know that there exists a sufficiently small $r>0$ such that

$$
\|T y\|>\|y\|, \quad \text { for any } y \in E \cap \partial \Omega_{r} .
$$

From $\left(\mathrm{H}_{6}\right)$ and the proof of Theorem 9, on the one hand, if $F^{0}=I^{0}=0$, we know that there exists a sufficiently small $r^{*}>0\left(r^{*}<r\right)$ such that

$$
\|T y\|<\|y\|, \quad \text { for any } y \in E \cap \partial \Omega_{r^{*}}
$$

In view of (67) and (68), by Lemma 2, it follows that $T$ has a fixed point $y^{*} \in E \cap\left(\Omega_{r} \backslash \overline{\Omega_{r^{*}}}\right)$ with $r^{*}<\left\|y^{*}\right\|<r$, which is a positive $\omega$-periodic solution of (1).

On the other hand, if $F^{\infty}=I^{\infty}=0$, we know that there exists a sufficiently large $R>0(R>r)$ such that

$$
\|T y\|<\|y\|, \quad \text { for any } y \in E \cap \partial \Omega_{R} \text {. }
$$

In view of (67) and (69), by Lemma 2 , it follows that $T$ has a fixed point $\bar{y} \in E \cap\left(\Omega_{R} \backslash \overline{\Omega_{r}}\right)$ with $r<\|\bar{y}\|<R$, which is a positive $\omega$-periodic solution of (1). Thus, (1) has at least two positive $\omega$-periodic solutions $y^{*}, \bar{y}$ satisfying $r^{*}<\left\|y^{*}\right\|<r<$ $\|\bar{y}\|<R$. The proof is completed.

Corollary 23. Assume that $\left(H_{1}\right)-\left(H_{4}\right),\left(H_{7}\right)$, and $\left(H_{13}\right)$ hold. Then, (1) has at least one positive $\omega$-periodic solution.

Similarly, one can prove the following theorems and corollaries.

Theorem 24. Assume that $\left(H_{1}\right)-\left(H_{4}\right),\left(H_{9}\right)$, and $\left(H_{11}\right)$ hold. Then, (1) has at least two positive $\omega$-periodic solutions $y^{*}, \bar{y}$ satisfying $0<\left\|y^{*}\right\|<\|\bar{y}\|$.

Corollary 25. Assume that $\left(H_{1}\right)-\left(H_{4}\right),\left(H_{10}\right)$, and $\left(H_{11}\right)$ hold. Then, (1) has at least one positive $\omega$-periodic solution.

Theorem 26. Assume that $\left(H_{1}\right)-\left(H_{4}\right),\left(H_{9}\right)$, and $\left(H_{14}\right)$ hold. Then, (1) has at least two positive $\omega$-periodic solutions $y^{*}, \bar{y}$ satisfying $0<\left\|y^{*}\right\|<\|\bar{y}\|$.

Corollary 27. Assume that $\left(H_{1}\right)-\left(H_{4}\right),\left(H_{10}\right)$, and $\left(H_{14}\right)$ hold. Then, (1) has at least one positive $\omega$-periodic solution. 


\section{Existence of Periodic Solution of (2)}

Now, we are in a position to attack the existence of positive periodic solutions of (2). By carrying out similar arguments as in Section 2, it is not difficult to establish sufficient criteria for the existence of positive periodic solutions of (2). For simplicity, we prefer to list below the corresponding criteria for (2) without proof, since the proofs are very similar to those in Section 2.

For $(t, s) \in R^{2}, 1 \leq i \leq n$, we define

$$
\begin{aligned}
G_{i}^{*}(t, s) & =\frac{e^{-\int_{t}^{s} a_{i}(\xi, y(\xi)) d \xi}}{1-e^{-\int_{0}^{\omega} a_{i}(\xi, y(\xi)) d \xi}} \\
& =\frac{e^{\int_{s}^{t+\omega}} a_{i}(\xi, y(\xi)) d \xi}{e^{\int_{0}^{\omega} a_{i}(\xi, y(\xi)) d \xi}-1}, \\
G^{*}(t, s) & =\operatorname{diag}\left[G_{1}^{*}(t, s), G_{2}^{*}(t, s), \ldots, G_{n}^{*}(t, s)\right] .
\end{aligned}
$$

It is clear that $G_{i}^{*}(t, t) \geq G_{i}^{*}(t, s) \geq G^{*}(t, t+\omega)$, $\partial G_{i}^{*}(t, s) /(\partial t)=a_{i}(t, y(t)) G_{i}^{*}(t, s), G_{i}^{*}(t, t)-G_{i}^{*}(t, t+\omega)=1$. In view of $\left(H_{1}\right)$, we also define for $1 \leq i \leq n$

$$
\begin{gathered}
\alpha_{i}^{*}:=\min _{0 \leq t \leq s \leq \omega}\left|G_{i}^{*}(t, s)\right|=\frac{1}{e^{\int_{0}^{\omega} a_{2 i}(\xi) d \xi}-1}=\alpha_{i}, \\
\beta_{i}^{*}:=\max _{0 \leq t \leq s \leq \omega}\left|G_{i}^{*}(t, s)\right|=\frac{e^{\int_{0}^{\omega} a_{2 i}(\xi) d \xi}}{e^{\int_{0}^{\omega} a_{1 i}(\xi) d \xi}-1}=\beta_{i}, \\
\alpha^{*}=\min _{1 \leq i \leq n} \alpha_{i}^{*}=\alpha, \quad \beta^{*}=\max _{1 \leq i \leq n} \beta_{i}^{*}=\beta, \\
\delta=\frac{\alpha^{*}}{\beta^{*}} \in(0,1)=\sigma, \\
B_{i}(t)=\max \left\{\left|b_{1 i}(t)\right|,\left|b_{2 i}(t)\right|\right\}, \\
B_{i}^{\prime}(t)=\min \left\{\left|b_{1 i}(t)\right|,\left|b_{2 i}(t)\right|\right\}, \\
B(t)=\max _{1 \leq i \leq n}\left\{B_{i}(t)\right\}, \\
B^{\prime}(t)=\min _{1 \leq i \leq n}\left\{B_{i}^{\prime}(t)\right\} .
\end{gathered}
$$

Let $X=\left\{y=\left(y_{1}(t), y_{2}(t), \ldots, y_{n}(t)\right)^{T} \in P C\left(R, R^{n}\right)\right.$ | $y(t+\omega)=y(t)\}$ with the norm $\|y\|=\sum_{i=1}^{n}\left|y_{i}\right|_{0},\left|y_{i}\right|_{0}=$ $\sup _{t \in[0, \omega]}\left|y_{i}(t)\right|$. It is easy to verify that $(X,\|\cdot\|)$ is a Banach space. Define $P$ as a cone in $X$ by

$$
\begin{gathered}
P=\left\{y=\left(y_{1}(t), y_{2}(t), \ldots, y_{n}(t)\right)^{T} \in X: y_{i}(t)\right. \\
\left.\geq \delta\left\|y_{i}\right\|_{0}, t \in[0, \omega]\right\} .
\end{gathered}
$$

We easily verify that $P$ is a cone in $X$. We define an operator $\phi: X \rightarrow X$ as follows:

$$
(\phi y)(t)=\left(\left(\phi_{1} y\right)(t),\left(\phi_{2} y\right)(t), \ldots,\left(\phi_{n} y\right)(t)\right)^{T},
$$

where

$$
\begin{aligned}
\left(\phi_{i} y\right)(t)=\lambda & \int_{t}^{t+\omega} G_{i}^{*}(t, s) b_{i}(s, y(s)) f_{i}(s, u(s)) d s \\
& +\mu \sum_{t \leq t_{k}<t+\omega} G_{i}^{*}\left(t, t_{k}\right) I_{i k}\left(t_{k}, y\left(t_{k}\right)\right) .
\end{aligned}
$$

The proof of the following lemmas, theorems, and corollaries is similar to those in Section 2; we omit all the details here.

Lemma 28. Assume that $\left(H_{1}\right)-\left(H_{4}\right)$ hold. The existence of positive $\omega$-periodic solution of (2) is equivalent to that of nonzero fixed point of $\phi$ in $P$.

Lemma 29. Assume that $\left(H_{1}\right)-\left(H_{4}\right)$ hold. Then $\phi: P \rightarrow P$ is well defined.

Lemma 30. Assume that $\left(H_{1}\right)-\left(H_{4}\right)$ hold. Then $\phi: P \rightarrow P$ is completely continuous.

Theorem 31. Assume $\left(H_{1}\right)-\left(H_{4}\right)$ and $\left(H_{5}\right)$ hold. Moreover, if one of the following conditions holds:

$$
\left(H_{6}\right) ;\left(H_{11}\right),\left(H_{14}\right)
$$

then, (2) has two positive $\omega$-periodic solutions $y^{*}$ and $\bar{y}$ satisfying $0<\left\|y^{*}\right\|<R<\|\bar{y}\|$, where $R$ is defined in $\left(H_{5}\right)$.

Theorem 32. Assume $\left(H_{1}\right)-\left(H_{4}\right)$ and $\left(H_{8}\right)$ hold. Moreover, if one of the following conditions holds:

$$
\left(H_{9}\right) ;\left(H_{12}\right),\left(H_{13}\right)
$$

then, (2) has two positive $\omega$-periodic solutions $y^{*}$ and $\bar{y}$ satisfying $0<\left\|y^{*}\right\|<r<\|\bar{y}\|$, where $r$ is defined in $\left(H_{8}\right)$.

Theorem 33. Assume $\left(H_{1}\right)-\left(H_{4}\right)$ hold. Moreover, if one of the following conditions holds:

$$
\begin{aligned}
& \left(H_{6}\right),\left(H_{12}\right) ;\left(H_{6}\right),\left(H_{13}\right) ; \\
& \left(H_{9}\right),\left(H_{11}\right) ;\left(H_{9}\right),\left(H_{14}\right),
\end{aligned}
$$

then, (2) has two positive $\omega$-periodic solutions $y^{*}$ and $\bar{y}$ satisfying $0<\left\|y^{*}\right\|<\|\bar{y}\|$.

Theorem 34. Assume $\left(H_{1}\right)-\left(H_{5}\right)$ hold. Moreover, if one of the following conditions holds:

$$
\left(H_{7}\right) ;\left(H_{8}\right) ;\left(H_{11}\right) ;\left(H_{14}\right)
$$

then, (2) has at least one positive $\omega$-periodic solution.

Theorem 35. Assume $\left(H_{1}\right)-\left(H_{4}\right)$ and $\left(H_{8}\right)$ hold. Moreover, if one of the following conditions holds:

$$
\left(H_{10}\right) ;\left(H_{12}\right) ;\left(H_{13}\right) \text {, }
$$

then, (2) has at least one positive w-periodic solution. 
Theorem 36. Assume $\left(H_{1}\right)-\left(H_{4}\right)$ hold. Moreover, if one of the following conditions holds:

$$
\begin{gathered}
\left(H_{7}\right),\left(H_{10}\right) ;\left(H_{7}\right),\left(H_{12}\right) ; \\
\left(H_{7}\right),\left(H_{13}\right) ;\left(H_{10}\right),\left(H_{11}\right) ; \\
\left(H_{10}\right),\left(H_{14}\right) ;\left(H_{11}\right),\left(H_{12}\right) ; \\
\left(H_{13}\right),\left(H_{14}\right),
\end{gathered}
$$

then, (2) has at least one positive $\omega$-periodic solution.

\section{Applications}

In this section, as some applications of our main results, we will consider some special cases of systems (1) and (2), which have been investigated extensively in the literature.

Application 1. Consider the following equations:

$$
\begin{aligned}
y^{\prime}(t)= & -A(t, y(t)) y(t) \\
& +\lambda B(t, y(t)) F(t, u(t)), \quad t \in R, \\
y^{\prime}(t)= & A(t, y(t)) y(t) \\
& -\lambda B(t, y(t)) F(t, u(t)), \quad t \in R,
\end{aligned}
$$

where $u(t)=\left(y\left(c_{1}(t)\right), \ldots, y\left(c_{n-1}(t)\right), \int_{-\infty}^{t} k(t-\xi) y(\xi) d \xi\right)$, which are special cases of systems (1) and (2) without impulse, respectively. First, we list several assumptions:

$$
\begin{aligned}
& \left(P_{1}\right)=\left(H_{1}\right) ; \\
& \left(P_{2}\right)=\left(H_{2}\right) ; \\
& \left(P_{3}\right)=\left(H_{3}\right) ; \\
& \left(P_{4}\right) \text { there exists a } R \quad>\quad 0 \text { such that } F(t, u) \geq \\
& \quad\left(R /\left(\alpha \sigma B^{\prime}(\xi) \lambda \omega\right)\right) \text { for }\|u\| \leq\|y\| \in[\sigma R, R] ; \\
& \left(P_{5}\right) \text { there exists a } r>0 \text { such that } F(t, u) \leq(r /(2 \beta B(\xi) \lambda \omega)) \\
& \quad \text { for }\|u\| \leq\|y\| \leq r ; \\
& \left(P_{6}\right) F^{0}=0 ; \\
& \left(P_{7}\right) F^{\infty}=0 ; \\
& \left(P_{8}\right) F_{0}=\infty ; \\
& \left(P_{9}\right) F_{\infty}=\infty ; \\
& \left(P_{10}\right) F^{0}=\eta_{1} \in(0,1 / \beta \lambda B(\xi) \omega) ; \\
& \left(P_{11}\right) F^{\infty}=\theta_{1} \in(0,1 / \beta \lambda B(\xi) \omega) ; \\
& \left(P_{12}\right) F_{0}=\eta_{2} \in\left(1 / \alpha \sigma \lambda B^{\prime}(\xi) \omega, \infty\right) ; \\
& \left(P_{13}\right) F_{\infty}=\theta_{2} \in\left(1 / \alpha \sigma \lambda B^{\prime}(\xi) \omega, \infty\right) .
\end{aligned}
$$

By applying theorems in Sections 2 and 3 and to systems (81) and (82), respectively, we obtain the following theorems.

Theorem 37. Assume $\left(P_{1}\right)-\left(P_{4}\right)$ hold. Moreover, if one of the following conditions holds:

$$
\left(P_{6}\right),\left(P_{7}\right) ;\left(P_{10}\right),\left(P_{11}\right),
$$

then, (81) and (82) have at least two positive w-periodic solutions.
Theorem 38. Assume $\left(P_{1}\right)-\left(P_{3}\right)$ and $\left(P_{5}\right)$ hold. Moreover, if one of the following conditions holds:

$$
\left(P_{8}\right),\left(P_{9}\right) ;\left(P_{12}\right),\left(P_{13}\right),
$$

then, (81) and (82) have at least two positive $\omega$-periodic solutions.

Theorem 39. Assume $\left(P_{1}\right)-\left(P_{3}\right)$ hold. Moreover, if one of the following conditions holds:

$$
\begin{aligned}
& \left(P_{6}\right),\left(P_{7}\right),\left(P_{12}\right) ;\left(P_{6}\right),\left(P_{7}\right),\left(P_{13}\right) ; \\
& \left(P_{8}\right),\left(P_{9}\right),\left(P_{10}\right) ;\left(P_{8}\right),\left(P_{9}\right),\left(P_{11}\right),
\end{aligned}
$$

then, (81) and (82) have at least two positive w-periodic solutions.

Theorem 40. Assume $\left(P_{1}\right)-\left(P_{4}\right)$ hold. Moreover, if one of the following conditions holds:

$$
\left(P_{5}\right) ;\left(P_{8}\right) ;\left(P_{9}\right) ;\left(P_{10}\right) ;\left(P_{11}\right),
$$

then, (81) and (82) have at least one positive w-periodic solution.

Theorem 41. Assume $\left(P_{1}\right)-\left(P_{3}\right)$ and $\left(P_{5}\right)$ hold. Moreover, if one of the following conditions holds:

$$
\left(P_{6}\right) ;\left(P_{7}\right) ;\left(P_{12}\right) ;\left(P_{13}\right),
$$

then (81) and (82) have at least one positive w-periodic solution.

Theorem 42. Assume $\left(P_{1}\right)-\left(P_{3}\right)$, hold. Moreover, if one of the following conditions holds:

$$
\begin{aligned}
& \left(P_{6}\right),\left(P_{9}\right) ;\left(P_{6}\right),\left(P_{13}\right) ; \\
& \left(P_{7}\right),\left(P_{8}\right) ;\left(P_{7}\right),\left(P_{12}\right) ; \\
& \left(P_{8}\right),\left(P_{11}\right) ;\left(P_{9}\right),\left(P_{10}\right) ; \\
& \left(P_{10}\right),\left(P_{13}\right) ;\left(P_{11}\right),\left(P_{12}\right),
\end{aligned}
$$

then, (81) and (82) have at least one positive w-periodic solution.

Application 2. Consider the following equations:

$$
\begin{array}{cl}
y^{\prime}(t)=-a(t) y(t)+f(t, u(t)), & t \in R, t \neq t_{k}, \\
\Delta y\left(t_{k}\right)=\mu I_{k}\left(t_{k}, y\left(t_{k}\right)\right), & k \in Z_{+}, \\
y^{\prime}(t)=a(t) y(t)-f(t, u(t)), & t \in R, t \neq t_{k}, \\
\Delta y\left(t_{k}\right)=\mu I_{k}\left(t_{k}, y\left(t_{k}\right)\right), & k \in Z_{+},
\end{array}
$$

where

$$
\begin{array}{r}
u(t)=\left(y\left(g_{1}(t)\right), \ldots, y\left(g_{n-1}(t)\right),\right. \\
\left.\int_{-\infty}^{t} k(t-\xi) y(\xi) d \xi\right),
\end{array}
$$


which are special cases of systems (1) and (2), respectively. For convenience in the following discussion, on the one hand, we introduce the following notations:

$$
\begin{aligned}
& f^{a}=\limsup _{u \in E,\|u\| \rightarrow a} \max _{t \in[0, \omega]} \frac{\int_{0}^{\omega}|f(t, u(t))| d t}{\|u\|}, \\
& f_{a}=\liminf _{u \in E,\|u\| \rightarrow a} \min _{t \in[0, \omega]} \frac{\int_{0}^{\omega}|f(t, u(t))| d t}{\|u\|}, \\
& I^{a}=\limsup _{y \in E,\|y\| \rightarrow a} \max _{t \in[0, \omega]} \frac{\sum_{t \leq t_{k}<t+\omega}\left|I_{k}(t, y)\right|}{\|y\|}, \\
& I_{a}=\liminf _{y \in E,\|y\| \rightarrow a} \min _{t \in[0, \omega]} \frac{\sum_{t \leq t_{k}<t+\omega}\left|I_{k}(t, y)\right|}{\|y\|},
\end{aligned}
$$

where $a$ denotes either 0 or $\infty,\|u\|=\max \left\{\left|u_{1}\right|,\left|u_{2}\right|, \ldots,\left|u_{n}\right|\right\}$. On the other hand, we list several assumptions:

$$
\begin{aligned}
& \left(A_{1}\right)=\left(H_{1}\right) ; \\
& \left(A_{2}\right)=\left(H_{2}\right) ; \\
& \left(A_{3}\right)=\left(H_{3}\right) ; \\
& \left(A_{4}\right)=\left(H_{4}\right) ; \\
& \left(A_{5}\right) \text { there exists a } R>0 \text { such that } f(t, u) \geq(R / 2 \alpha \sigma \lambda \omega), \\
& \quad \sum_{t \leq t_{k}<t+\omega} H_{k}\left(t_{k}, y\left(t_{k}\right)\right) \geq(R / 2 \alpha \sigma \mu), \text { for }\|u\| \leq\|y\| \in \\
& \quad[\sigma R, R] ; \\
& \left(A_{6}\right) \text { there exists a } r>0 \text { such that } f(t, u) \leq(r / 2 \beta \lambda \omega), \\
& \quad \sum_{t \leq t_{k}<t+\omega} I\left(t_{k}, y\left(t_{k}\right)\right) \leq(r / 2 \beta \mu), \text { for }\|u\| \leq\|y\| \leq r ; \\
& \left(A_{7}\right) f^{0}=I^{0}=0 ; \\
& \left(A_{8}\right) f^{\infty}=I^{\infty}=0 ; \\
& \left(A_{9}\right) f_{0}=I_{0}=\infty ; \\
& \left(A_{10}\right) f_{\infty}=I_{\infty}=\infty ; \\
& \left(A_{11}\right) f^{0}=\rho_{1} \in(0,1 / 2 \beta \lambda \omega), I^{0}=\xi_{1} \in(0,1 / 2 \beta \mu) ; \\
& \left(A_{12}\right) f^{\infty}=\varrho_{1} \in(0,1 / 2 \beta \lambda \omega), I^{\infty}=\varsigma_{1} \in(0,1 / 2 \beta \mu) ; \\
& \left(A_{13}\right) f_{0}=\rho_{2} \in(1 / 2 \alpha \sigma \lambda \omega, \infty), I_{0}=\xi_{2} \in(1 / 2 \alpha \sigma \mu, \infty) ; \\
& \left(A_{14}\right) f_{\infty}=\varrho_{2} \in(1 / 2 \alpha \sigma \lambda \omega, \infty), I_{\infty}=\varsigma_{2} \in(1 / 2 \alpha \sigma \mu, \infty) .
\end{aligned}
$$

By applying theorems in Sections 2 and 3 and to systems (89) and (90), respectively, we obtain the following theorems.

Theorem 43. Assume $\left(A_{1}\right)-\left(A_{5}\right)$ hold. Moreover, if one of the following conditions holds:

$$
\left(A_{7}\right),\left(A_{8}\right) ;\left(A_{11}\right),\left(A_{12}\right),
$$

then, (89) and (90) have at least two positive $\omega$-periodic solutions.

Theorem 44. Assume $\left(A_{1}\right)-\left(A_{4}\right)$ and $\left(A_{6}\right)$ hold. Moreover, if one of the following conditions holds:

$$
\left(A_{9}\right),\left(A_{10}\right) ;\left(A_{13}\right),\left(A_{14}\right)
$$

then, (89) and (90) have at least two positive w-periodic solutions.
Theorem 45. Assume $\left(A_{1}\right)-\left(A_{4}\right)$ hold. Moreover, if one of the following conditions holds:

$$
\begin{aligned}
& \left(A_{7}\right),\left(A_{8}\right),\left(A_{13}\right) ;\left(A_{7}\right),\left(A_{8}\right),\left(A_{14}\right) ; \\
& \left(A_{9}\right),\left(A_{10}\right),\left(A_{11}\right) ;\left(A_{9}\right),\left(A_{10}\right),\left(A_{12}\right),
\end{aligned}
$$

then, (89) and (90) have at least two positive $\omega$-periodic solutions.

Theorem 46. Assume $\left(A_{1}\right)-\left(A_{5}\right)$ hold. Moreover, if one of the following conditions holds:

$$
\left(A_{5}\right) ;\left(A_{9}\right) ;\left(A_{10}\right) ;\left(A_{11}\right) ;\left(A_{12}\right)
$$

then, (89) and (90) have at least one positive $\omega$-periodic solution.

Theorem 47. Assume $\left(A_{1}\right)-\left(A_{4}\right)$ and $\left(A_{6}\right)$ hold. Moreover, if one of the following conditions holds:

$$
\left(A_{7}\right) ;\left(A_{8}\right) ;\left(A_{13}\right) ;\left(A_{14}\right) \text {, }
$$

then, (89) and (90) have at least one positive $\omega$-periodic solution.

Theorem 48. Assume $\left(A_{1}\right)-\left(A_{4}\right)$ hold. Moreover, if one of the following conditions holds:

$$
\begin{aligned}
& \left(A_{7}\right),\left(A_{10}\right) ;\left(A_{7}\right),\left(A_{14}\right) ; \\
& \left(A_{8}\right),\left(A_{9}\right) ;\left(A_{8}\right),\left(A_{13}\right) ; \\
& \left(A_{9}\right),\left(A_{12}\right) ;\left(A_{10}\right),\left(A_{11}\right) ; \\
& \left(A_{11}\right),\left(A_{14}\right) ;\left(A_{12}\right),\left(A_{13}\right),
\end{aligned}
$$

then, (89) and (90) have at least one positive $\omega$-periodic solution.

Application 3. Consider the following equations:

$$
\begin{array}{ll}
y^{\prime}(t)=-a(t) y(t)+f(t, u(t)), & t \in R, \\
y^{\prime}(t)=a(t) y(t)-f(t, u(t)), & t \in R,
\end{array}
$$

where

$$
\begin{array}{r}
u(t)=\left(y\left(c_{1}(t)\right), \ldots, y\left(c_{n-1}(t)\right),\right. \\
\left.\int_{-\infty}^{t} k(t-\xi) y(\xi) d \xi\right),
\end{array}
$$

which are special cases of systems (89) and (90) without impulse, respectively, that is, $I\left(t_{k}, y\left(t_{k}\right)\right)=0$. For convenience in the following discussion, we list several assumptions:

$$
\begin{aligned}
& \left(A_{1}^{*}\right)=\left(H_{1}\right) ; \\
& \left(A_{2}^{*}\right)=\left(H_{2}\right) ; \\
& \left(A_{3}^{*}\right)=\left(H_{3}\right) ;
\end{aligned}
$$


$\left(A_{4}^{*}\right)$ there exists a $R>0$ such that $f(t, u) \geq(R / 2 \alpha \sigma \lambda \omega)$, for $\|u\| \leq\|y\| \in[\sigma R, R]$

$\left(A_{5}^{*}\right)$ there exists a $r>0$ such that $f(t, u) \leq(r / 2 \beta \lambda \omega)$, for $\|u\| \leq\|y\| \leq r$

$$
\begin{aligned}
& \left(A_{6}^{*}\right) f^{0}=0 ;\left(A_{7}^{*}\right) f^{\infty}=0 ; \\
& \left(A_{8}^{*}\right) f_{0}=\infty ;\left(A_{9}^{*}\right) f_{\infty}=\infty ; \\
& \left(A_{10}^{*}\right) f^{0}=\rho_{1} \in(0,1 /(2 \beta \lambda \omega)) ; \\
& \left(A_{11}^{*}\right) f^{\infty}=\varrho_{1} \in(0,1 /(2 \beta \lambda \omega)) ; \\
& \left(A_{12}^{*}\right) f_{0}=\rho_{2} \in(1 /(2 \alpha \sigma \lambda \omega, \infty)) ; \\
& \left(A_{13}^{*}\right) f_{\infty}=\varrho_{2} \in(1 /(2 \alpha \sigma \lambda \omega, \infty)) .
\end{aligned}
$$

We obtain the following theorems.

Corollary 49. Assume $\left(A_{1}^{*}\right)-\left(A_{4}^{*}\right)$ hold. Moreover, if one of the following conditions holds:

$$
\left(A_{6}^{*}\right),\left(A_{7}^{*}\right) ;\left(A_{10}^{*}\right),\left(A_{11}^{*}\right),
$$

then, (99) and (100) have at least two positive w-periodic solutions.

Corollary 50. Assume $\left(A_{1}^{*}\right)-\left(A_{3}^{*}\right)$ and $\left(A_{5}^{*}\right)$ hold. Moreover, if one of the following conditions holds:

$$
\left(A_{8}^{*}\right),\left(A_{9}^{*}\right) ;\left(A_{12}^{*}\right),\left(A_{13}^{*}\right),
$$

then, (99) and (100) have at least two positive $\omega$-periodic solutions.

Corollary 51. Assume $\left(A_{1}^{*}\right)-\left(A_{3}^{*}\right)$ hold. Moreover, if one of the following conditions holds:

$$
\begin{aligned}
& \left(A_{6}^{*}\right),\left(A_{7}^{*}\right),\left(A_{12}^{*}\right) ;\left(A_{6}^{*}\right),\left(A_{7}^{*}\right),\left(A_{13}^{*}\right) ; \\
& \left(A_{8}^{*}\right),\left(A_{9}^{*}\right),\left(A_{10}^{*}\right) ;\left(A_{8}^{*}\right),\left(A_{9}^{*}\right),\left(A_{11}^{*}\right),
\end{aligned}
$$

then, (99) and (100) have at least two positive w-periodic solutions.

Corollary 52. Assume $\left(A_{1}^{*}\right)-\left(A_{4}^{*}\right)$ hold. Moreover, if one of the following conditions holds:

$$
\left(A_{5}^{*}\right) ;\left(A_{8}^{*}\right) ;\left(A_{9}^{*}\right) ;\left(A_{10}^{*}\right) ;\left(A_{11}^{*}\right),
$$

then, (99) and (100) have at least one positive w-periodic solution.

Corollary 53. Assume $\left(A_{1}^{*}\right)-\left(A_{3}^{*}\right),\left(A_{5}^{*}\right)$ hold. Moreover, if one of the following conditions holds:

$$
\left(A_{6}^{*}\right) ;\left(A_{7}^{*}\right) ;\left(A_{12}^{*}\right) ;\left(A_{13}^{*}\right),
$$

then, (99) and (100) have at least one positive $\omega$-periodic solution.
Corollary 54. Assume $\left(A_{1}^{*}\right)-\left(A_{3}^{*}\right)$ hold. Moreover, if one of the following conditions holds:

$$
\begin{gathered}
\left(A_{6}^{*}\right),\left(A_{9}^{*}\right) ;\left(A_{6}^{*}\right),\left(A_{13}^{*}\right) ; \\
\left(A_{7}^{*}\right),\left(A_{8}^{*}\right) ;\left(A_{7}^{*}\right),\left(A_{12}^{*}\right) ; \\
\left(A_{8}^{*}\right),\left(A_{11}^{*}\right) ;\left(A_{9}^{*}\right),\left(A_{10}^{*}\right) ; \\
\left(A_{10}^{*}\right),\left(A_{13}^{*}\right) ;\left(A_{11}^{*}\right),\left(A_{12}^{*}\right),
\end{gathered}
$$

then, (99) and (100) have at least one positive $\omega$-periodic solution.

Hence, our results generalize and improve the corresponding results of [16].

Application 4. Consider the generalized logistic model of single species $[16,21]$ with impulse and two parameters:

$$
\begin{aligned}
& y^{\prime}(t)=y(t) a(t) \\
& -\lambda y(t)\left[\sum_{i=1}^{n} b_{i}(t) y\left(t-\tau_{i}(t)\right)\right. \\
& \left.\quad+c(t) \int_{-\infty}^{t} k(t-s) y(s) d s\right], \\
& \quad t \in R, t \neq t_{k}, \\
& \Delta y\left(t_{k}\right)=\mu I_{k}\left(t_{k}, y\left(t_{k}\right)\right), \quad k \in Z_{+},
\end{aligned}
$$

where $a(t), b_{i}(t), \tau_{i}(t), c(t) \in C(R, R+)$ are $\omega$-periodic, $\lambda>0$, $\mu>0$ are two parameters, and $k: R_{+} \rightarrow R_{+}$is integrable such that $\int_{0}^{\omega} k(t) d t=1$.

Theorem 55. Assume that $I_{k} \in C\left(R, R_{-}\right)$and the following conditions hold:

(1) $I^{0}=0, I_{\infty}=\infty$;

(2) $\sum_{i=1}^{n} b_{i}^{L}+c^{L}>0$;

then, (108) has at least one positive $\omega$-periodic solution, where

$$
\begin{gathered}
\alpha=\frac{1}{e^{\int_{0}^{\omega} a(t) d t}-1}, \quad \beta=\frac{e^{\int_{0}^{\omega} a(t) d t}}{e^{\int_{0}^{\omega} a(t) d t}-1}, \\
\sigma=\frac{\alpha}{\beta}, \quad g^{M}=\max _{t \in[0, \omega]} g(t), \quad g^{L}=\min _{t \in[0, \omega]} g(t) .
\end{gathered}
$$

Proof. We let

$$
\begin{gathered}
f(t, u(t))=u_{0}\left[\sum_{i=1}^{n} b_{i}(t) u_{i}(t)+c(t) u_{n+1}\right], \\
u=\left(u_{0}, u_{1}, \ldots, u_{n}, u_{n+1}\right) \in R^{n+2} .
\end{gathered}
$$


Then, (108) can be seen as a special form of (2) satisfying $\left(H_{1}\right)-\left(H_{4}\right)$. We can construct the same Banach space $X$ and cones $\mathrm{E}$ as in Section 2. Then for any $y \in E$,

$$
\begin{aligned}
& \int_{0}^{\omega}|f(t, u(t))| d t \\
& \quad=\int_{0}^{\omega}\left|u_{0}\left[\sum_{i=1}^{n} b_{i}(t) u_{i}+c(t) u_{n+1}\right]\right| d t \\
& \geq \sigma\|u\|^{2}\left[\sum_{i=1}^{n} b_{i}^{L}+c^{L}\right] \omega
\end{aligned}
$$

so we have

$$
\frac{\int_{0}^{\omega}|f(t, u(t))| d t}{\|u\|} \longrightarrow \infty, \quad\|u\| \longrightarrow \infty ;
$$

that is, $f_{\infty}=\infty$. On the other hand,

$$
\begin{aligned}
\int_{0}^{\omega}|f(t, u(t))| d t \\
\quad=\int_{0}^{\omega}\left|u_{0}\left[\sum_{i=1}^{n} b_{i}(t) u_{i}+c(t) u_{n+1}\right]\right| d t \\
\quad \leq\|u\|^{2}\left[\sum_{i=1}^{n} b_{i}^{M}+c^{M}\right] \omega,
\end{aligned}
$$

which can lead to

$$
\frac{\int_{0}^{\omega}|f(t, u(t))| d t}{\|u\|} \longrightarrow 0, \quad\|u\| \longrightarrow 0
$$

that is, $f^{0}=0$. Then in view of (2), (112), and (114), we can obtain that (108) has at least one positive $\omega$-periodic solution. The proof is complete.

Application 5. Consider the generalized so-called Nicholson's Blowflies model $[19,20]$ with impulse and two parameters:

$$
\begin{aligned}
y^{\prime}(t)= & -y(t) a(t) \\
& +\lambda b(t) y(t-\tau(t)) e^{-c(t) y(t-\tau(t))}, \\
& t \in R, t \neq t_{k}, \\
\Delta y\left(t_{k}\right) & =\mu I_{k}\left(t_{k}, y\left(t_{k}\right)\right), \quad k \in Z_{+},
\end{aligned}
$$

where $a(t), b(t), c(t) \tau(t) \in C(R, R+)$ are $\omega$-periodic and $\lambda>$ $0, \mu>0$ are two parameters.

Theorem 56. Assume that $I_{k} \in C\left(R, R_{-}\right)$and the following conditions hold:

(3) $I^{0}=\infty, I_{\infty}=0$;

then, (115) has at least one positive $\omega$-periodic solution, where

$$
\begin{gathered}
\eta=\frac{1}{e^{\int_{0}^{\omega} a(t) d t}-1}, \quad \theta=\frac{e^{\int_{0}^{\omega} a(t) d t}}{e^{\int_{0}^{\omega} a(t) d t}-1}, \\
\delta=\frac{\eta}{\theta}, \quad g^{M}=\max _{t \in[0, \omega]} g(t), \quad g^{L}=\min _{t \in[0, \omega]} g(t) .
\end{gathered}
$$

Proof. We let $f(t, u(t))=b(t) u(t) e^{-c(t) u(t)}$. Then, (115) can be seen as a special form of (1) satisfying $\left(H_{1}\right)-\left(H_{4}\right)$. We can construct the same Banach space $X$ and cones $P$ as in Section 2. Then for any $y \in P$,

$$
\begin{aligned}
\int_{0}^{\omega}|f(t, u(t))| d t \\
\quad=\int_{0}^{\omega}\left|b(t) u(t) e^{-c(t) u(t)}\right| d t \\
\quad \geq\|u\| \frac{b^{L}}{e^{c^{M}\|u\|}} \omega .
\end{aligned}
$$

So we have

$$
\frac{\int_{0}^{\omega}|f(t, u(t))| d t}{\|u\|} \longrightarrow 0, \quad\|u\| \longrightarrow \infty ;
$$

that is, $f_{\infty}=0$. On the other hand,

$$
\begin{aligned}
& \int_{0}^{\omega}|f(t, u(t))| d t \int_{0}^{\omega}\left|b(t) u(t) e^{-c(t) u(t)}\right| d t \\
& \quad \leq\|u\| \frac{b^{M}}{e^{c^{L}\|u\|}} \omega,
\end{aligned}
$$

which can lead to

$$
\frac{\int_{0}^{\omega}|f(t, u(t))| d t}{\|u\|} \longrightarrow \infty, \quad\|u\| \longrightarrow 0 ;
$$

that is, $f^{0}=\infty$. Then in view of (1), (93), and (120), we can obtain that (115) has at least one positive $\omega$-periodic solution. The proof is complete.

\section{Conflict of Interests}

The authors declare that there is no conflict of interests regarding the publication of this paper.

\section{Acknowledgments}

This research is supported by NSF of China (nos. 10971229, 11161015, 11371367, and 11361012), PSF of China (nos. 2012M512162 and 2013T60934), NSF of Hunan Province (nos. 11JJ900, 12JJ9001, and 13JJ4098), the Education Foundation of Hunan Province (nos. 12C0541 and 13C084), the Science Foundation of Hengyang Normal University (no. 11B36), and the construct program of the key discipline in Hunan Province.

\section{References}

[1] V. Lakshmikantham, D. D. Baĭnov, and P. S. Simeonov, Theory of Impulsive Differential Equations, vol. 6, World Scientific, Singapore, 1989.

[2] D. D. Bainov and P. S. Simeonov, Impulsive Differential Equations: Periodic Solutions and Applications, Longman Scientific and Technical, 1993. 
[3] D. D. Bainov and P. S. Simeonov, Impulsive Differential Equations, vol. 28, World Scientific, Singapore, 1995.

[4] J. R. Yan and A. M. Zhao, "Oscillation and stability of linear impulsive delay differential equations," Journal of Mathematical Analysis and Applications, vol. 227, no. 1, pp. 187-194, 1998.

[5] G. Ballinger and X. Liu, "Existence, uniqueness and boundedness results for impulsive delay differential equations," Applicable Analysis, vol. 74, no. 1-2, pp. 71-93, 2000.

[6] J. J. Nieto, "Impulsive resonance periodic problems of first order," Applied Mathematics Letters, vol. 15, no. 4, pp. 489-493, 2002.

[7] Z. G. Luo, B. X. Dai, and Q. Wang, "Existence of positive periodic solutions for a nonautonomous neutral delay $n$-species competitive model with impulses," Nonlinear Analysis: Real World Applications, vol. 11, no. 5, pp. 3955-3967, 2010.

[8] X. Y. Li, X. N. Lin, D. Q. Jiang, and X. Y. Zhang, "Existence and multiplicity of positive periodic solutions to functional differential equations with impulse effects," Nonlinear Analysis: Theory, Methods and Applications, vol. 62, no. 4, pp. 683-701, 2005.

[9] Z. G. Luo and L. P. Luo, "Global positive periodic solutions of generalized $\mathrm{n}$-species competition systems with multiple delays and impulses," Abstract and Applied Analysis, vol. 2013, Article ID 980974, 12 pages, 2013.

[10] M. Liu and K. Wang, "Asymptotic behavior of a stochastic nonautonomous Lotka-Volterra competitive system with impulsive perturbations," Mathematical and Computer Modelling, vol. 57, no. 3-4, pp. 909-925, 2013.

[11] J. R. Yan, "Global attractivity for impulsive population dynamics with delay arguments," Nonlinear Analysis: Theory, Methods and Applications, vol. 71, no. 11, pp. 5417-5426, 2009.

[12] Y. Kuang, Delay Differential Equations with Applications in Population Dynamics, vol. 191, Academic Press, Boston, Mass, USA, 1993.

[13] M. Fan and K. Wang, "Optimal harvesting policy for single population with periodic coefficients," Mathematical Biosciences, vol. 152, no. 2, pp. 165-177, 1998.

[14] A. Y. Wan and D. Q. Jiang, "Existence of positive periodic solutions for functional differential equations," Kyushu Journal of Mathematics, vol. 56, no. 1, pp. 193-202, 2002.

[15] H. Y. Wang, "Positive periodic solutions of functional differential equations," Journal of Differential Equations, vol. 202, no. 2, pp. 354-366, 2004.

[16] D. Ye, M. Fan, and H. Y. Wang, "Periodic solutions for scalar functional differential equations," Nonlinear Analysis: Theory, Methods and Applications, vol. 62, no. 7, pp. 1157-1181, 2005.

[17] J. R. Yan, "Existence of positive periodic solutions of impulsive functional differential equations with two parameters," Journal of Mathematical Analysis and Applications, vol. 327, no. 2, pp. 854-868, 2007.

[18] Z. J. Zeng, B. Li, and M. Fan, "Existence of multiple positive periodic solutions for functional differential equations," Journal of Mathematical Analysis and Applications, vol. 325, no. 2, pp. 1378-1389, 2007.

[19] M. Wazewska-Czyzewska and A. Lasota, "Mathematical models of the red cell system," Matematyka Stosowana, vol. 6, pp. 23-40, 1976.

[20] W. S. Gurney, S. P. Blythe, and R. M. Nisbet, "Nicholson's blowflies revisited," Nature, vol. 287, pp. 17-21, 1980.

[21] Y. K. Li, "Existence and global attractivity of positive periodic solutions for a class ofdelay differential equations," Chinese Science Bulletin, vol. 28, no. 2, pp. 108-118, 1998.
[22] D. J. Guo, Nonlinear Functional Analysis, ShanDong Science and Technology Press, 2001.

[23] K. Deimling, Nonlinear Functional Analysis, Springer, Berlin, Germany, 1985.

[24] M. A. Krasnoselskii, Positive Solution of Operator Equation, P. Noordhoff, Groningen, The Netherlands, 1964.

[25] D. J. Guo and V. Lakshmikantham, Nonlinear Problems in Abstract Cones, vol. 5, Academic Press, Orlando, Fla, USA, 1988.

[26] N. Zhang, B. X. Dai, and X. Z. Qian, "Periodic solutions for a class of higher-dimension functional differential equations with impulses," Nonlinear Analysis: Theory, Methods and Applications, vol. 68, no. 3, pp. 629-638, 2008.

[27] Y. H. Fan, W. T. Li, and L. L. Wang, "periodic solutions of delayed ratio-dependent predator-prey models with monotonic or monotonic or nonmonotonic functional responses," Nonlinear Analysis: Real World Applications, vol. 5, no. 2, pp. 247-263, 2004. 


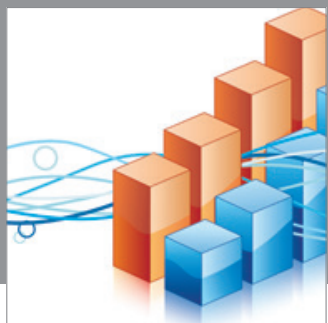

Advances in

Operations Research

mansans

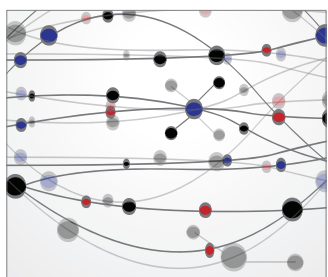

The Scientific World Journal
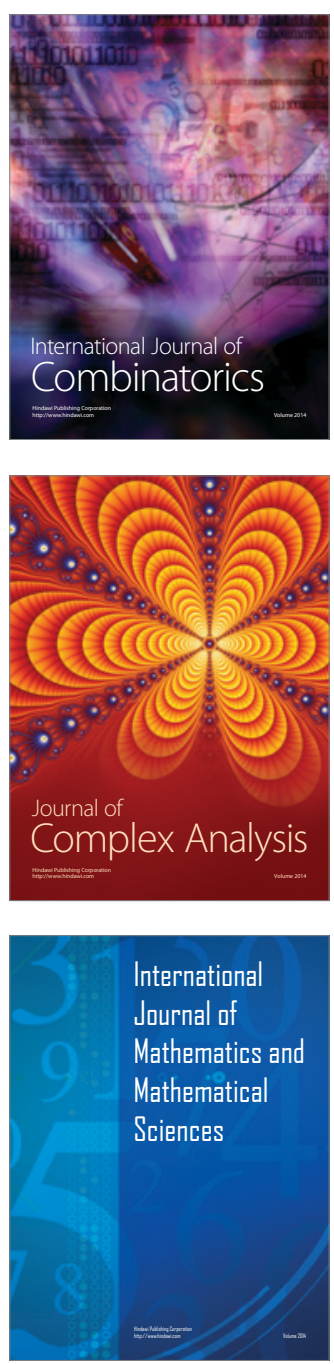
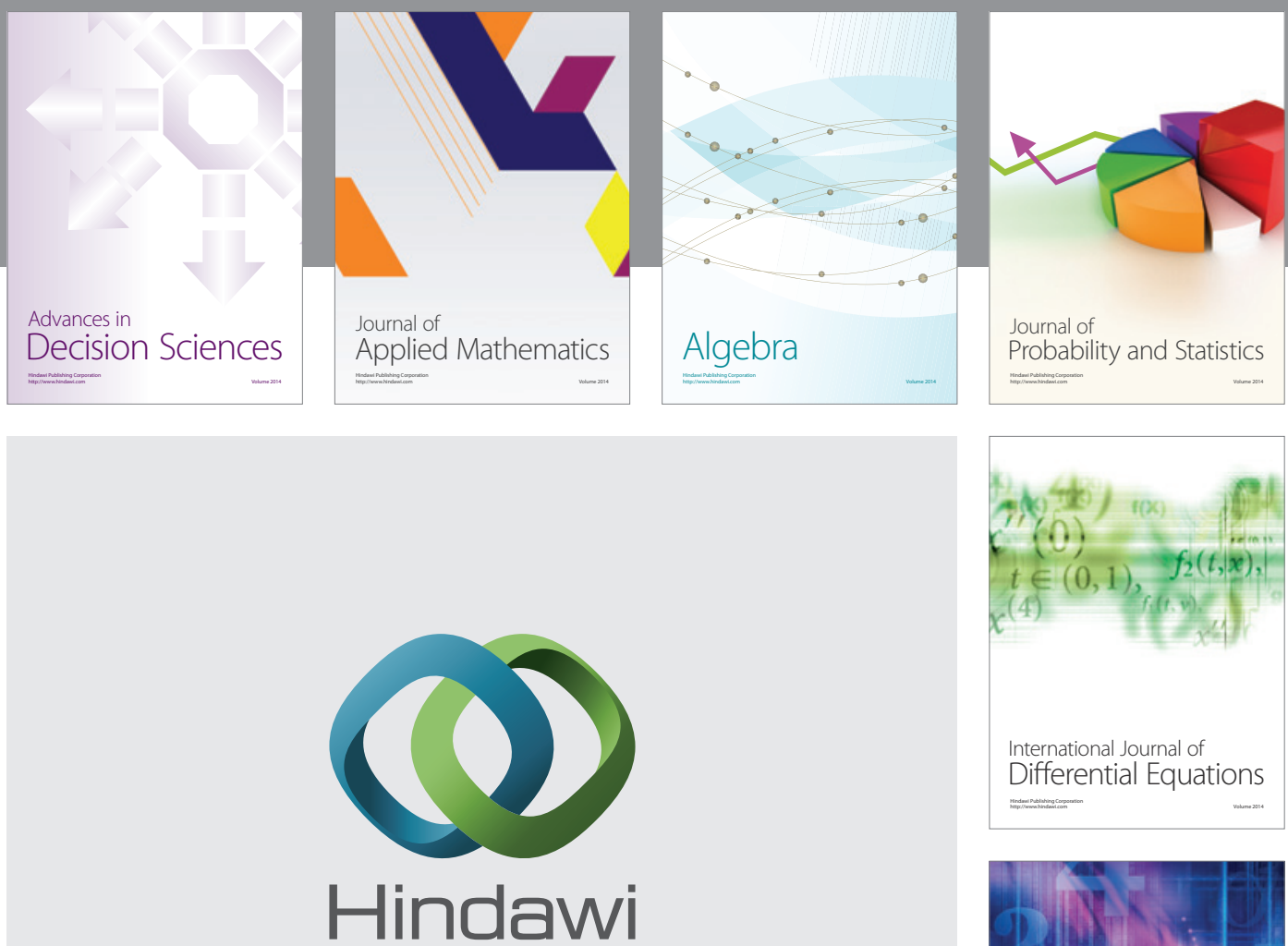

Submit your manuscripts at http://www.hindawi.com
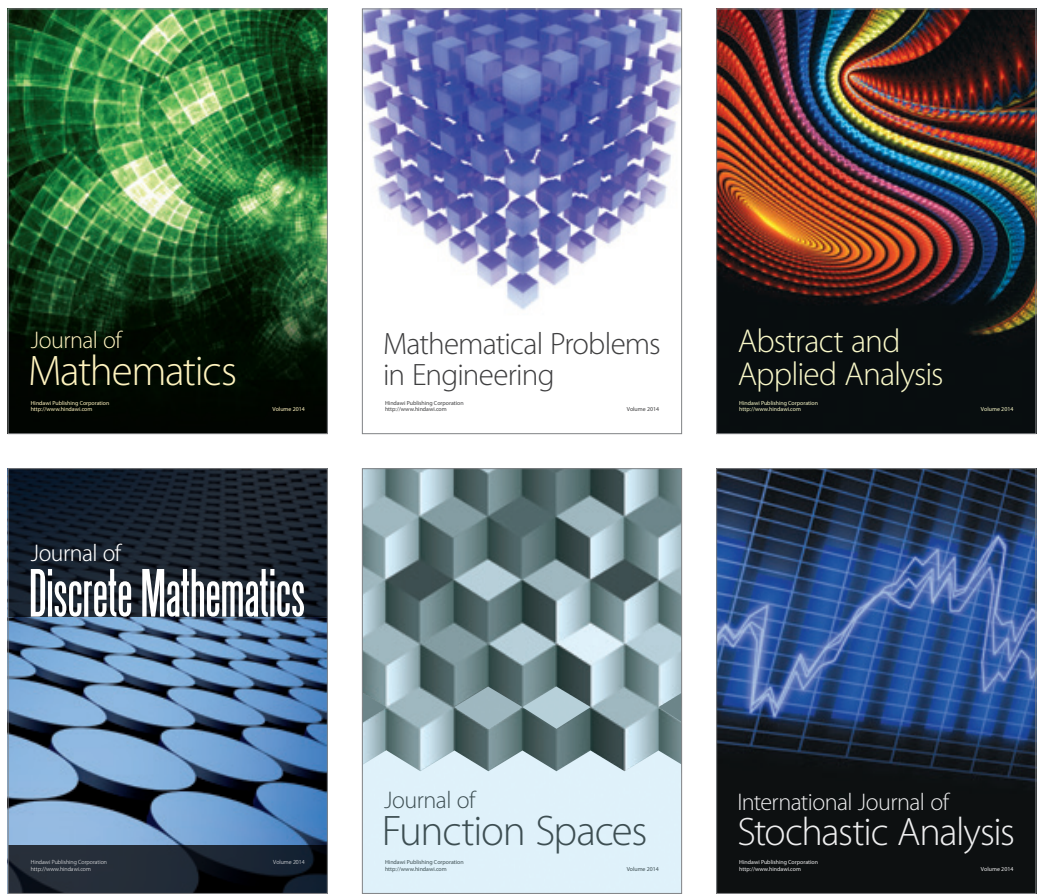

Journal of

Function Spaces

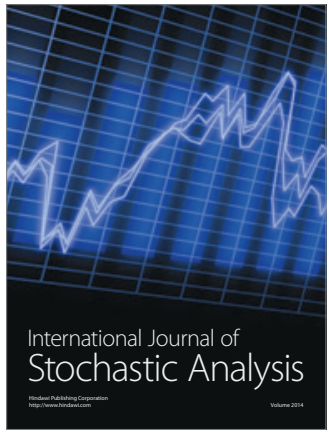

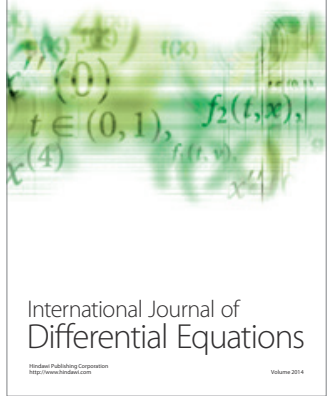
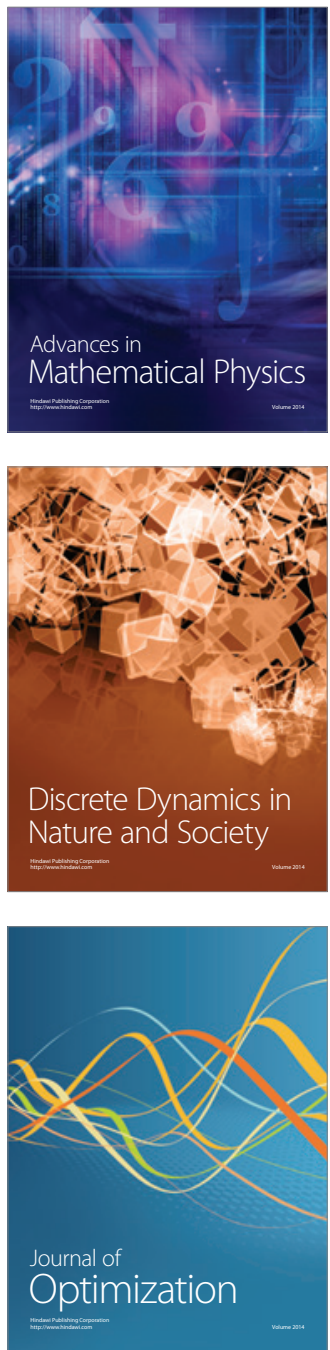